\title{
Multi-particle scattering and breakdown of the Wiedemann-Franz law at a junction of $N$ interacting quantum wires
}

\author{
Domenico Giuliano, $, 2, *$ Andrea Nava,,${ }^{1,2}$ Reinhold Egger,${ }^{3}$ Pasquale Sodano, ${ }^{4}$ and Francesco Buccheri ${ }^{3, \dagger}$ \\ ${ }^{1}$ Dipartimento di Fisica, Università della Calabria, Arcavacata di Rende I-87036, Cosenza, Italy \\ ${ }^{2}$ I.N.F.N. - Gruppo collegato di Cosenza, Arcavacata di Rende I-87036, Cosenza, Italy \\ ${ }^{3}$ Institut für Theoretische Physik, Heinrich-Heine Universität, D-40225 Düsseldorf, Germany \\ ${ }^{4}$ I.N.F.N., Sezione di Perugia, Via A. Pascoli, I-06123, Perugia, Italy
}

(Dated: January 24, 2022)

\begin{abstract}
We analyze the charge and thermal transport at a junction of interacting quantum wires close to equilibrium. Within the framework of Tomonaga-Luttinger liquids, we compute the thermal conductance for a wide class of boundary conditions and detail the physical processes leading to the breakdown of the Wiedemann-Franz law at the junction. We show how connecting external reservoirs to the quantum wires affects the conductance tensors close to the various fixed points of the phase diagram of the junction. We therefore distinguish two types of violation of the WiedemannFranz law: a "trivial" one, independent of the junction dynamics and arising from the breakdown of the Fermi-liquid picture in the wire, and a junction-related counterpart, arising from multi-particle scattering processes at the junction.
\end{abstract}

\section{INTRODUCTION}

Junctions of interacting quantum wires (QWs), realized with both spinless [1-5], or spinful systems [6-8], have continuously attracted the attention of physicists, in that they can be regarded as the simplest components of a quantum circuit. Furthermore, a plethora of unconventional phases can be realized at a pertinently engineered junction, which correspond to attractive fixed points in the boundary phase diagram of the system at which Landau's Fermi liquid paradigm breaks down. There are indeed various reasons for the emergence of non Fermi liquid phases, related to the peculiar nature of the elementary excitations in effectively one-dimensional interacting electronic systems, to the topology of the junction, or to the dynamics of localized excitations emerging at the junction itself. In fact, the low-lying elementary excitations in an interacting fermionic system in one dimension are not particles and holes, but instead collective bosonic modes, whose dynamics is encoded in the Tomonaga-Luttinger liquid paradigm [9 and 10]. The loss of integrity of particle and hole excitations formally corresponds to the description of tunneling processes at the junction in terms of operators that are nonlinear functionals of the Tomonaga-Luttinger liquid fields. The corresponding scaling dimensions continuously depend on the "bulk" interaction [11 and 12], which allows for stabilizing, for instance, non Fermi liquid phases with, e.g., "fractional" tunneling of excitations with charge, but without spin, and vice versa [13 and 14]. Also, the onset of multiparticle scattering processes [15] gives rise to non Fermi liquid stable phases at strong enough values of the interaction in three-wire junctions, both in the spinless [2], as well as in the spinful [6], case.

Stabilizing non-Fermi liquid phases typically requires un physically high values of the bulk interaction strength, which gives rise to relevant operators destabilizing the Fermi liquid phase(s) and, at the same time, stabilizing the non-Fermi liquid ones. Alternatively, at small values of the bulk interaction, or even in the noninteracting limit, the onset of non Fermi liquid phases may be determined by the interaction between the collective modes of the leads and local degrees of freedom emerging at the junction.

In particular, due to the high versatility of the Tomonaga-Luttinger liquid approach in describing onedimensional spin chains in the spin-liquid phase [16], as well as one-dimensional Josephson junction arrays [17 and 18], or pertinently engineered cold atom systems [19], (Kondo-like) models of local magnetic impurities interacting with the collective excitations of the leads have been proposed in junctions of spin chains [20-23], of one-dimensional Josephson junction networks [24 and 25], of cold atom condensates [26]. At variance, local fermionic degrees of freedom can emerge as Klein factors, that is, real fermion operators required on implementing the bosonization over a junction with more than two leads [2 and 22]. Finally, localized degrees of freedom are clearly present when the leads are proximity-coupled at one end to a central island, i.e., a mesoscopic system, either grounded or floating, with a finite charging energy. The number of degrees of freedom is typically limited due to the physical size, as in quantum dots, or by a gapped spectrum, which suppresses the excitations at low temperatures. A remarkable example is provided by localized Majorana zero modes (MZMs) in junctions involving superconducting islands [27-29]. On entangling with the lead degrees of freedom, or with each other, Klein factors and MZMs trigger the onset of nontrivial phases and/or phase transitions in junctions of QWs, for many of which a full theoretical description is still lacking, or applicable only to leading order in the distance (in parameter space) from other phases for which there is a complete theoretical model [2, 6, 30-33].

An efficient mean to identify the system phases is by looking at the equilibrium charge and energy transport properties of the system under investigation (see, for in- 
stance, [34] for a review). These are typically not independent of each other: whenever an electronic system can be adiabatically deformed into a noninteracting Fermi gas, the ratio between the charge and the thermal conductance of the system are related by the WiedemannFranz law (WFL). This states that, at temperatures low with respect to the Fermi energy, such ratio is proportional to the temperature, through a universal constant $L_{0}$, dubbed Lorenz number [35]

$$
L_{0}=\frac{\pi^{2} k_{B}^{2}}{3 e^{2}} \approx 2.44 \times 10^{-8} W \Omega K^{-2} .
$$

In practice, the WFL is experimentally well-verified in metals and semiconductors at room temperature [36], though interaction effects and inelastic scattering may lead to a renormalization of the Lorenz number [37-39]. Also, localized interactions, e.g., such as in quantum dots, may provide an analogous renormalization of $L_{0}$ by a nonuniversal value [40-42].

It is worth mentioning that the WFL is expected to be satisfied when a one-dimensional system is connected to external Fermi liquid reservoirs [2, 43-45]. In the same way, one does not expect deviations of the Lorenz number from $L_{0}$ in the presence of the Kondo effect, in the co-tunneling regime of an interacting quantum dot, due to the emergence of Nozierès Fermi liquid phase [4648]. In interacting quantum wires, the results are analogous [49]. Somewhat surprisingly, the WFL appears to be valid even in a non Fermi liquid phase, such as for the overscreened Kondo fixed point [50-52]. As long as charge and heat are carried by the same excitations, regardless of whether they are Landau quasiparticles, or collective modes of the Tomonaga-Luttinger liquid leads, the Lorenz ratio remains unchanged [53].

A phase (a fixed point) of the junction is uniquely identified by the local relations between the fields describing the collective excitations in the wires. Typically, these are recovered by looking at the (equilibrium) transport properties of the system (electrical conductance for charged wires, spin conductance for spin liquids, et cetera) $[2,30$, and 54]. However, when comparing the theoretical predictions with the experimental results, in many cases of physical interest this procedure may be tainted by spurious effects, that may spoil the interpretation of the measurements. Therefore, to unambiguously identify the phases setting in at junctions of QWs, it becomes important to make combined measurements of the charge transport properties of the system and of additional, pertinently chosen, quantities.

In this paper, we investigate the charge and the energy transport through a junction of $N>2$ interacting QWs. In doing so, we are able to determine under which conditions and by means of which physical mechanisms the WFL is violated at the junction. Physically, this takes place when the charge-carrying excitations are separated from the heat-carrying ones. Consistently, we show that the WFL is expected to break down whenever Andreev reflection and/or crossed Andreev reflection pro- cesses take place at the junction, possibly in combination with the normal reflection and the normal transmission of particles. The coexistence of normal and Andreev reflection/transmission makes the net charge flowing in one direction different from the net number of particles flowing in the same direction, thus leading to a remarkable "charge-heat separation". Based on this observation, we go through an analysis of the charge and transport conductances across a junction of $N$ QWs, both in the case of noninteracting and of interacting leads. We find that the WFL is violated only if the charge is not conserved at the junction, which typically requires coupling the QWs to an underneath superconductor. Typically, in this case, the Lorenz number is rescaled by a nonuniversal factor, which depends on the microscopic scattering processes at the central island [55]. Conversely, we show that the WFL breaks down also with charge conservation holding at the junction when the low-energy modes of the leads are coupled to MZMs localized at the central island. The interaction allows for stabilizing phases characterized by multiparticle normal and Andreev reflection/transmission processes at the central island, at a fixed, universal and predictable renormalization of the Lorenz ratio. Beside being genuine non Fermi liquid phases, due to the multiparticle scattering processes being the only ones that survive in the zero-temperature limit, these phases feature a remarkable charge-heat separation [56], with the consequent breakdown of the WFL, in an universal and predictable way.

Importantly, in order to recover the breakdown of the WFL in a junction of normal wires, such as the ones studied in [2 and 6], we need to have an extremely strong bulk interaction in the wire. Alternatively, coupling the wires with emerging MZMs at the central island can effectively stabilize the breakdown of the WFL at relatively small interactions, or even with noninteracting leads [57]. The formalism we develop here, based on the TomonagaLuttinger liquid approach, allows us to make sharp predictions on the charge and thermal conductance tensors at the low-temperature fixed point (phase), as well as on their scaling dependence on the temperature in its vicinity. As an example, we extend the calculation in [57] for a junction providing a realization of the topological Kondo model (TKM) [27, 28, and 58].

Within our analysis, we also discuss in detail the modifications induced in the various phases and, more in general, in the whole topology of the phase diagram, when the junction is connected (as it is typical, in transport measurements) to outer, Fermi liquid reservoirs. When the reservoirs are attached at a (finite) distance $\ell$ from the junction, at low enough energies/long enough wavelengths, the system becomes sensitive to their presence. Specifically, this implies that, when considering the equilibrium (dc) charge and thermal conductances, the effects related to the bulk interaction in the leads, including the renormalization of the Lorenz ratio, are eventually washed out by the presence of the effectively noninteracting reservoirs [2, 43-45]. Often, in transport measure- 
ments, one has to consider the effects of the reservoirs. Therefore, we devote part of our work to disentangle the violation of the WFL determined by the bulk interaction in the leads from the one genuinely due to the onset of multiparticle (anomalous) scattering processes at the junction. In doing so, we also argue how the reservoirs affect the scaling of the corrections to the fixed point values of the conductances and of the Lorenz ratio and how they may modify the phase diagram itself. Based on the results of this paper, in [57] we conclude that the emergence of MZMs and their action in inducing the topological Kondo effect becomes the effective mechanism triggering the (universal) breakdown of the WFL at the junction and that such phenomenon can be exploited for detection of the presence of MZMs at the junction. While standard charge transport experiments cannot presently distinguish MZMs from other effects unambiguously [5963], our proposal provides a new experimental test, aimed at ruling out such ambiguities.

The paper is organized as follows:

- In Section II, we derive the charge conductance tensor and the thermal conductance tensor at a junction of $N$ one-dimensional, noninteracting, spinless QWs and we evidence the importance of Andreev and/or crossed Andreev reflection at the junction, in order for the WFL to break down.

- In Section III, we set up the formalism to compute the charge and the thermal conductance tensor within the Tomonaga-Luttinger liquid approach. In particular, we trace out a direct correspondence between the conformal boundary conditions describing a fixed point in the phase diagram of the junction and the corresponding charge and heat conductance tensors.

- In Section IV, we compute the charge and the thermal conductance tensor at various fixed points of a generic $N$-wire junction. We evidence the violations of the WFL and discuss the two mechanisms that originate it.

- In Section V, we discuss the WFL in a junction of $N=3$ QWs [1 and 2] and in the TKM [27, 28 , and 58]. In reviewing the phase diagram of both systems, we also derive the scaling properties (with the temperature as scaling parameter) of the conductance tensors and of the Lorenz ratio close to each fixed point. We analyze both the disconnected case and the one in which the junction is connected to external reservoirs.

- In Section VI we provide our conclusions and discuss about possible further developments of our work.

- In the various Appendices we provide mathematical details of our work.

\begin{tabular}{|c|c|}
\hline QW & Quantum wire \\
\hline MZM & Majorana zero mode \\
\hline WFL & Wiedemann-Franz law \\
\hline TKM & Topological Kondo model \\
\hline CCT & Charge conductance tensor \\
\hline HCT & Heat conductance tensor \\
\hline (D)FP & (Disconnected) Fixed point \\
\hline RG & Renormalization group \\
\hline
\end{tabular}

Table I. Glossary of most commonly used abbreviations

To help following the various abbreviations, we list in table I the meaning of the ones we use most commonly throughout the paper.

\section{CHARGE AND THERMAL CONDUCTANCE TENSOR AT A JUNCTION OF NONINTERACTING QUANTUM WIRES}

We now derive the charge conductance tensor (CCT) and the heat conductance tensor (HCT) at a junction of $N$ one-dimensional, noninteracting, spinless QWs, connected to each other at $x=0$. In the following we limit our analysis to the case in which the wires are all equal to each other, though this implies no loss of generality in our derivation. As we focus on the equilibrium transport properties of the junction, we resort to a low-energy, long-wavelength expansion around the Fermi points, so to write the junction Hamiltonian as $H=H_{\mathrm{Fer}, 0}+H_{B}$. With $H_{B}$ we denote the boundary Hamiltonian encoding the system dynamics at the junction, while the "lead" Hamiltonian $H_{\mathrm{Fer}, 0}$ is given by

$$
H_{\mathrm{Fer}, 0}=-i v \sum_{j=1}^{N} \int_{0}^{\ell} d x\left\{\psi_{R, j}^{\dagger} \partial_{x} \psi_{R, j}-\psi_{L, j}^{\dagger} \partial_{x} \psi_{L, j}\right\}
$$

In Eq.(2) we denote with $\psi_{R / L, j}$ the chiral fermionic fields corresponding to the two chiral excitation branches around the Fermi points, with the subscripts $R(L)$ labeling right-handed (left-handed) branches and $v$ the Fermi velocity. Also, we introduce the lead length $\ell$ as a large-distance regularization. Eventually, when computing physical quantities, we take the $\ell \rightarrow \infty$ limit.

To encompass also the case of junctions involving superconductors, we consider all the possible single-particle scattering processes, which we draw in Fig.1. Specifically, we see that an incoming $L$ electron from wire $j$ can be reflected within the same wire as an electron ("normal reflection"), or reflected within the same wire as a hole ("Andreev reflection"), or transmitted to wire $j^{\prime}(\neq j)$ as an electron ("normal transmission"), or, finally, transmitted to wire $j^{\prime}$ as a hole ("crossed Andreev reflection"). Denoting respectively with $r_{j^{\prime}, j^{\prime}}, a_{j^{\prime}, j^{\prime}}, t_{j^{\prime}, j}, c_{j^{\prime}, j}$ the corresponding scattering amplitudes, we encode them in the 


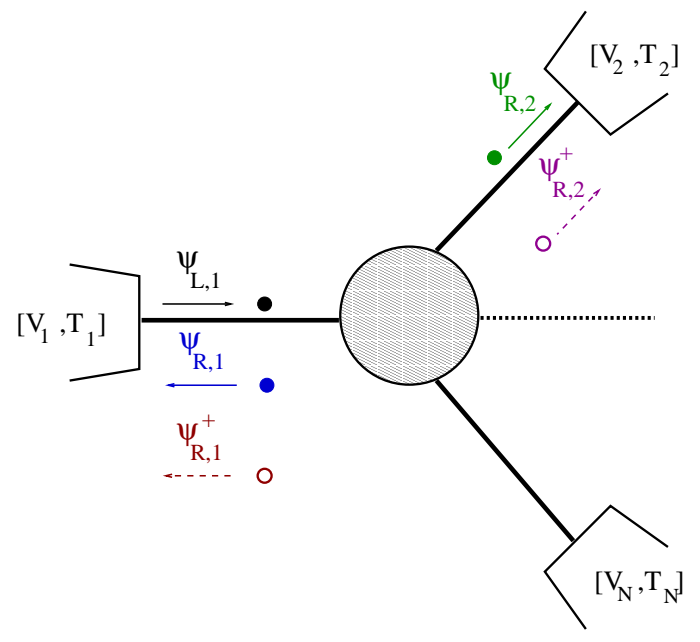

Figure 1. Sketch of the fermionic $N$ wire junction. Chiral fermions are shot toward the central island from the external reservoirs, biased at voltage and temperature $V_{j}, T_{j}$, with $j=$ $1, \ldots, N$. An incoming $L$ electron from wire $j^{\prime}(=1$ in the figure) (black dot) can be reflected within the same wire as an electron ("normal reflection", blue dot), or reflected within the same wire as a hole ("Andreev reflection", red empty dot), or transmitted to wire $j\left(\neq j^{\prime},=2\right.$ in the figure $)$ as an electron ("normal transmission", green dot), or, finally, transmitted to wire $j$ as a hole ("crossed Andreev reflection", magenta empty dot).

"extended" $2 N \times 2 N$ S matrix

$$
\begin{aligned}
& \psi_{R, j^{\prime}}(0)=\sum_{j=1}^{N}\left\{S_{j^{\prime}, j} \psi_{L, j}(0)+S_{j^{\prime}, j+N} \psi_{L, j}^{\dagger}(0)\right\} \\
& \psi_{R, j^{\prime}}^{\dagger}(0)=\sum_{j=1}^{N}\left\{S_{j^{\prime}+N, j} \psi_{L, j}(0)+S_{j^{\prime}+N, j+N} \psi_{L, j}^{\dagger}(0)\right\} .
\end{aligned}
$$

In Eq.(3) we have labeled the $(2 N \times 2 N) \mathbf{S}$ matrix so that, in the matrix elements $S_{a, b}$, indices $a(b)=1, \ldots, N$ refer to particles, indices $a(b)=N+1, \ldots, 2 N$ refer to holes. Assuming, as we do throughout all our paper, that particle-hole symmetry holds in our system, we infer that the $\mathbf{S}$ matrix must satisfy the Bogoliubov-de Gennes constraint [33], $\mathbf{S}^{\dagger}=K_{C} \mathbf{S} K_{C}$, with $K_{C}=\sigma^{x} \otimes \mathbf{I}_{N}$, where the Pauli matrix $\sigma^{x}$ acts in particle-hole space. This implies (assuming $j^{\prime}=1, \ldots, N$ )

$$
\begin{aligned}
S_{j^{\prime}, j} & =\delta_{j^{\prime}, j} r_{j^{\prime}, j^{\prime}}+\left[1-\delta_{j^{\prime}, j}\right] t_{j^{\prime}, j} \\
S_{j^{\prime}, j+N} & =\delta_{j^{\prime}, j} a_{j^{\prime}, j^{\prime}}+\left[1-\delta_{j^{\prime}, j}\right] c_{j^{\prime}, j} \\
S_{j^{\prime}+N, j} & =S_{j^{\prime}, j+N}^{*} \\
S_{j^{\prime}+N, j+N} & =S_{j^{\prime}, j}^{*} .
\end{aligned}
$$

Going through a similar derivation, we recover the $\mathbf{S}$ matrix encoding the scattering of an incoming hole from lead $j$ throughout the wires connected to each other at the central island (see Appendix A for details). Keeping only the linearly dispersing low-energy modes, the $\mathbf{S}$ matrix elements in Eqs.(3) become independent of the energy. While such an approximation does not affect our following derivation, we refer to Appendix A for a full discussion of the scattering amplitudes within a lattice fermionic model for the leads, which allows to retain the full energy dependence of the $\mathbf{S}$ matrix at any step of the derivation of the conductance tensors.

Various proposals have recently been formulated, about realizing junctions exhibiting either Andreev, or crossed Andreev reflection, or both. For instance, it has been pointed out that Andreev reflection and crossed Andreev reflection can become relevant processes in junctions realized by depositing quantum wires on top of a superconducting island with finite charging energy $E_{c}$ and Josephson coupling to a superconductor $[27,28,58,64$, and 65]. In this case, Andreev reflection and crossed Andreev reflection are triggered by the emergence of localized MZMs [27, 28, and 66], thus giving rise to a remarkable "topological" Kondo effect. A similar physics is expected to arise at a junction between several quantum wires, or a multichannel quantum wire, and a topological superconductor [31-33]. More generically, Andreev reflection and/or crossed Andreev reflection can arise at junctions of normal quantum wires, but at the cost of having a strong electronic interaction within each wire [2 and 6]. Finally, we note that nonlocal crossed Andreev reflection can be in principle recovered across a finite-size one-dimensional topological superconductor with long range pairing and/or electron hopping [67 and $68]$.

Here, given the extended $\mathbf{S}$ matrix at the junction, we now compute the equilibrium $\mathrm{CCT}$ and $\mathrm{HCT}$ of a junction connected to external reservoirs, such as the one we sketch in Fig.1. We regard the external reservoirs as noninteracting Fermi liquids, each one characterized by a voltage bias $V_{j}$ and by a temperature $T_{j}$. Paraphrasing [69], we describe each of them by means of the Fermi distribution functions for a single particle-like eigenmode at energy $\epsilon, f_{j}^{(p)}(\epsilon)$, and for a hole-like eigenmode at energy $\epsilon, f_{j}^{(h)}(\epsilon)$. The distribution functions are respectively given by

$$
\begin{aligned}
f_{j}^{(p)}(\epsilon) & =\frac{1}{1+e^{\beta_{j}\left(\epsilon-\mu-e V_{j}\right)}} \\
f_{j}^{(h)}(\epsilon) & =\frac{1}{1+e^{\beta_{j}\left(\epsilon-\mu+e V_{j}\right)}},
\end{aligned}
$$

with $\mu$ being the common reference (for all the leads) chemical potential and $\beta_{j}=\left[k_{B}\left(T+\delta T_{j}\right)\right]^{-1}$, with $T$ being the common reference temperature. Of course, in order for linear response theory (which we employ in the following) to apply, we require that $\left|e V_{j} / \mu\right|,\left|\delta T_{j} / T\right| \ll 1$, $\forall j=1, \ldots, N$. In order to compute the CCT and the $\mathrm{HCT}$, in the following we look at the average values of the electric current density operator in lead $j, j_{\mathrm{el}, j}$ and of the energy current density operator in lead $j, j_{\mathrm{th}, j}$. These can be readily recovered by means of the appropriate continuity equations for the electric charge density operator and for the energy density operator. They are given by 


$$
\begin{aligned}
& j_{\mathrm{el}, j}(x, t)=e v\left\{\psi_{R, j}^{\dagger} \psi_{R, j}-\psi_{L, j}^{\dagger} \psi_{L, j}\right\} \\
& j_{\mathrm{th}, j}(x, t)=-i v^{2}\left\{\psi_{R, j}^{\dagger} \partial_{x} \psi_{R, j}+\psi_{L, j}^{\dagger} \partial_{x} \psi_{L, j}\right\} .
\end{aligned}
$$

Denoting with $I_{\mathrm{el}, j}$ and with $I_{\mathrm{th}, j}$ the expectation values of the operators in Eqs.(6), as detailed in Appendix A, we obtain the CCT and the HCT matrix elements

$$
\begin{aligned}
G_{j, j^{\prime}} & =\frac{e^{2}}{2 \pi}\left(-\delta_{j, j^{\prime}}+T_{j, j^{\prime}}-A_{j, j^{\prime}}\right) \\
K_{j, j^{\prime}} & =\frac{\pi k_{B}^{2} T}{6}\left(-\delta_{j, j^{\prime}}+T_{j, j^{\prime}}+A_{j, j^{\prime}}\right),
\end{aligned}
$$

with $T_{j, j^{\prime}}=\left|t_{j, j^{\prime}}\right|^{2}$ if $j \neq j^{\prime}$, while $T_{j, j}=\left|r_{j}\right|^{2}$, as well as $A_{j, j^{\prime}}=\left|c_{j, j^{\prime}}\right|^{2}$ if $j \neq j^{\prime}$, while $A_{j, j}=\left|a_{j}\right|^{2}$. The thermal current obeys the Kirchhoff law [56], from which

$$
\sum_{j^{\prime}=1}^{N}\left\{T_{j, j^{\prime}}+A_{j, j^{\prime}}\right\}=\sum_{j^{\prime}=1}^{N}\left\{T_{j^{\prime}, j}+A_{j^{\prime}, j}\right\}=1,
$$

$\forall j=1, \ldots, N$. This is a direct consequence of the unitarity of the extended S-matrix.

If the charge is conserved at the junction, then Kirchhoff laws holds for the electric current as well and $A_{j^{\prime}, j}=0, \forall j, j^{\prime}$ necessarily. In a junction of multiple QWs, we modify the temperature or the electrochemical potential in one of the reservoirs and measure the charge or heat current that exits the junction in another wire. Therefore, a natural extension of the definition of Lorenz ratio in our geometry is [56]

$$
\mathcal{L}_{j, j^{\prime}}=\frac{K_{j, j^{\prime}}}{T G_{j, j^{\prime}}},
$$

and using Eqs.(7), we readily obtain the WFL $K_{j, j^{\prime}}=T L_{0} G_{j, j^{\prime}}$, with $L_{0}$ in Eq.(1). Eq.(9) is defined only when the denominator is non-zero. In the opposite case, for $j, j^{\prime}$ such that $G_{j, j^{\prime}}=0$, the correct result is obtained via perturbation theory, which will be addressed in sections III and V. Instead, if the junction does not conserve the electric charge, as in the presence of a grounded superconductor, we do not expect the Kirchhoff law for the electric current to hold. We therefore obtain

$$
\mathcal{L}_{j, j^{\prime}}=\frac{T_{j, j^{\prime}}+A_{j, j^{\prime}}-\delta_{j, j^{\prime}}}{T_{j, j^{\prime}}-A_{j, j^{\prime}}-\delta_{j, j^{\prime}}} L_{0},
$$

Let us consider Eq.(10) for $j \neq j^{\prime}$ : we see that, while the contributions to the thermal conductance by normal transmission and crossed Andreev reflection have the same sign, the ones to the electric conductance have opposite sign, which, in general, implies a Lorenz ratio $\mathcal{L}>L_{0}$ as soon as $A_{j, j^{\prime}}>0$. This is a consequence of the fact that outgoing particles and holes move in the same directions, but with opposite charges. Therefore, while the energy currents carried by the two of them add up, the electric currents get subtracted from each other. Similarly, considering the ratios between the diagonal conductances in Eq.(10), we see that normal reflection and Andreev reflection both lower the thermal current within lead $j$, while the former lowers and the latter increases the electric current. In conclusion, we have established that, whenever only single-particle scattering processes take place at the central island (as it typically happens when, e.g., the leads are noninteracting) and if the total charge is conserved, no violation of the WFL can be realized at a junction of $N$ quantum wires. This conclusion can be circumvented by having a nonzero interaction within the leads and/or by physically relevant mechanisms, which can stabilize nontrivial phases of the system characterized by multi-particle scattering processes at the junction. In order to study a number of system in which this takes place, we now generalize our derivation to the case of interacting leads, which requires to resort to the framework of Abelian bosonization for one-dimensional systems.

\section{TRANSPORT AT A JUNCTION OF INTERACTING QUANTUM WIRES AND CONFORMAL BOUNDARY CONDITIONS}

We now set up the formalism to compute the charge and the thermal conductance tensor at a junction of interacting ballistic QWs within the Luttinger liquid approach of $[13,14,37$, and 38]. On one hand, this allows us to account for a nonzero bulk interaction in the leads, on the other hand, it enables us to generate boundary conditions different from the one arising from single-particle scattering discussed in Section II.

\section{A. Electric and thermal conductance}

Within the Tomonaga-Luttinger liquid approach, the chiral fermionic operators in each lead are realized as functionals of the plasmon fields $\phi_{j}(x)$, together with their conjugates $\theta_{j}(x)$, satisfying the commutation relations $\left[\phi_{j}(x), \theta_{j^{\prime}}\left(x^{\prime}\right)\right]=\frac{i}{2} \delta_{j, j^{\prime}} \epsilon\left(x-x^{\prime}\right)$, with $\epsilon\left(x-x^{\prime}\right)$ being the sign function, with all the other commutators being zero. Specifically, they are represented as the vertex operators

$$
\begin{aligned}
& \psi_{R, j}(x)=\Gamma_{j} e^{i \sqrt{\pi}\left[\phi_{j}(x)+\theta_{j}(x)\right]} \\
& \psi_{L, j}(x)=\Gamma_{j} e^{i \sqrt{\pi}\left[\phi_{j}(x)-\theta_{j}(x)\right]},
\end{aligned}
$$

with the Klein factors $\Gamma_{j}$ being real fermion operators, satisfying the algebra $\left\{\Gamma_{j}, \Gamma_{j^{\prime}}\right\}=2 \delta_{j, j^{\prime}}$, introduced in order to assure the appropriate anticommutation relations between single electron annihilation/creation operators acting over different leads. The dynamics of the lead bosonic fields is encoded in the Hamiltonian $H_{0, \text { Bos }}$, given by

$$
H_{0, \mathrm{Bos}}=\frac{u}{2} \sum_{j=1}^{N} \int_{0}^{\ell} d x\left[g\left(\partial_{x} \phi_{j}(x)\right)^{2}+g^{-1}\left(\partial_{x} \theta_{j}(x)\right)^{2}\right],
$$


with $u$ being the collective plasmon velocity and $g$ being the dimensionless Luttinger parameter (again, for the sake of simplicity, we assume that all the wires are characterized by the same parameters $g$ and $u$. Yet, our derivation can be readily extended to a junction of Luttinger liquids with different parameters by a pertinent implementation of, e.g., the methods developed in [6 and 31]), complemented with the appropriate boundary conditions at the "inner" boundary $x=0$. In terms of the fields $\phi_{j}$ and $\theta_{j}$, the charge density and the charge current operators in lead $j, \rho_{\mathrm{el}, j}(x, t), j_{\mathrm{el}, j}(x, t)$, are given by

$$
\begin{aligned}
\rho_{\mathrm{el}, j}(x, t) & =\frac{e}{\sqrt{\pi}} \partial_{x} \phi_{j}(x, t) \\
j_{\mathrm{el}, j}(x, t) & =\frac{e u g}{\sqrt{\pi}} \partial_{x} \theta_{j}(x, t),
\end{aligned}
$$

and are related to each other via a continuity equation. In the same way, the energy current $j_{\mathrm{en}, j}$ can be defined from the continuity equation for the Hamiltonian density in (12) as

$$
j_{\mathrm{en}, j}(x, t)=u^{2} \partial_{x} \phi_{j}(x, t) \partial_{x} \theta_{j}(x, t) \quad .
$$

The heat current $j_{\mathrm{th}, j}$ follows from the above expression as $j_{\mathrm{th}, j}=j_{\mathrm{en}, j}-V_{j} j_{\mathrm{el}, j}[56]$. In fact, at charge neutrality, the energy and thermal currents yield the same results for the conductances in the linear response regime.

Working with ballistic QWs, in the following we will see that the CCT and the HCT are only affected by the scattering processes at the central island. While our approach is effective in working out the zero-temperature, fixed point properties of the junction, in general other effects, which we do not consider here, such as coupling with phonons, may become effective in determining the finite-temperature transport properties of our system [70] (see, e.g., [39 and 71] for a comprehensive discussion of several possible physical mechanisms affecting the thermal transport properties of an electronic system).

In Fig.2 we provide a sketch of our junction: within a generalization of the calculation for a single wire discussed in [37 and 38], the QW $j$ is connected to an external reservoir that injects chiral, left-handed modes, at chemical potential $V_{j}$ and temperature $T_{j}$. Accordingly, we introduce the chiral bosonic fields in the lead $j, \varphi_{R, j}(x), \varphi_{L, j}(x)$ : they are related to the fields $\phi_{j}(x), \theta_{j}(x)$ in Eq.(12) via the relations

$$
\begin{aligned}
\phi_{j}(x) & =\frac{\varphi_{R, j}(x)+\varphi_{L, j}(x)}{\sqrt{g}} \\
\theta_{j}(x) & =\sqrt{g}\left\{\varphi_{R, j}(x)-\varphi_{L, j}(x)\right\} .
\end{aligned}
$$

We describe the junction by means of pertinent conformal boundary conditions between the bosonic fields: in particular, the relation between the $R$ and the $L$ fields is encoded in the $N \times N$ splitting matrix $\rho$ [2,72, and 73]

$$
\varphi_{R, j}(0)=\sum_{j^{\prime}=1}^{N} \rho_{j, j^{\prime}} \varphi_{L, j^{\prime}}(0) .
$$

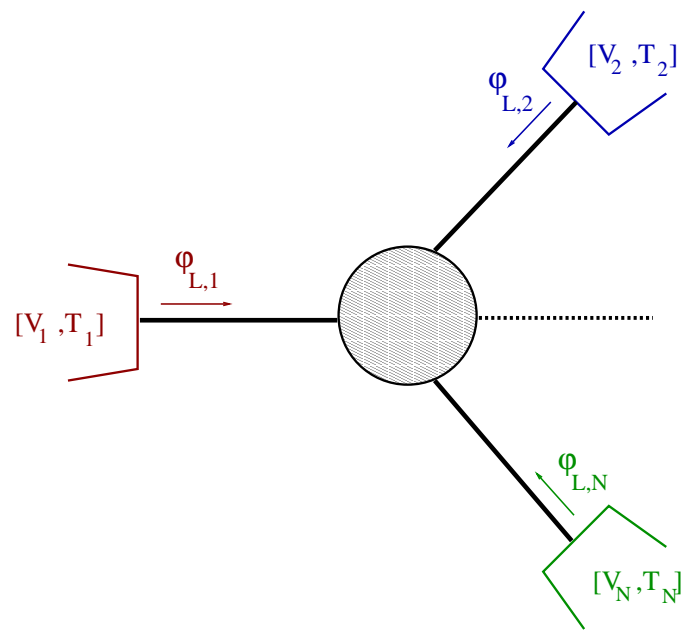

Figure 2. Sketch of a junction of $N$ interacting quantum wires. Each wire $j$ is connected to a reservoir, which injects into the system left-handed modes at voltage bias $V_{j}$ and at temperature $T_{j}$. The dashed region represents the central island, whose dynamics is encoded in the matrix $\rho$, relating the right-handed to the left-handed chiral modes at $x=0$.

By requiring that Eq.(16) is consistent with the canonical commutation relations between the $\varphi_{R, j}$ and the $\varphi_{L, j}$ fields, one readily finds that $\rho$ must be an orthogonal matrix. Typically, relations such as the ones in Eq.(16) hold at a conformally invariant fixed point of the phase diagram of the junction, where scale invariance implies that the splitting matrix does not depend on the momenta. Using Eq.(16), we define $N$ "unfolded" fields

$$
\varphi_{j}(x)=\left\{\begin{array}{l}
\varphi_{L, j}(x), \quad(0 \leq x \leq \ell) \\
\sum_{j^{\prime}=1}^{N} \rho_{j^{\prime}, j} \varphi_{R, j^{\prime}}(-x), \quad(-\ell \leq x<0) .
\end{array} .\right.
$$

By construction, each field $\varphi_{j}(x)$ is at chemical and thermal equilibrium with the reservoir at voltage bias $V_{j}$ and at temperature $T_{j}$. Accordingly, at nonzero biases, we rewrite the lead Hamiltonian in terms of the unfolded fields as $H_{0, \mathrm{Bos}}=\sum_{j=1}^{N} H_{0, \mathrm{Bos}, j}$, with

$$
H_{0, \mathrm{Bos}, j}=\int_{-\ell}^{\ell} d x\left\{u\left(\partial_{x} \varphi_{j}(x)\right)^{2}+e \sqrt{\frac{g}{\pi}} V_{j} \partial_{x} \varphi_{j}(x)\right\}
$$

Thus, once a generic observable $\mathcal{O}$ is expressed in terms of the fields $\varphi_{j}$, we compute its thermal average as

$$
\langle\mathcal{O}\rangle=\frac{\operatorname{Tr}\left[\mathcal{O} e^{-\sum_{j=1}^{N} \beta_{j} H_{0, \mathrm{Bos}, j}}\right]}{\prod_{j=1}^{N} \mathcal{Z}_{j}\left[V_{j}, \beta_{j}\right]},
$$

with $\beta_{j}=\left(k_{B} T_{j}\right)^{-1}$ and

$$
\mathcal{Z}_{j}\left[V_{j}, \beta_{j}\right]=\operatorname{Tr}\left[e^{-\beta_{j} H_{0, \mathrm{Bos}, j}}\right] \quad .
$$

In terms of the chiral fields the electric and thermal cur- 
rent operators in lead $j$ are

$$
\begin{aligned}
& j_{\mathrm{el}, j}(x, t)=e u \sqrt{\frac{g}{\pi}}\left[\partial_{x} \varphi_{j}\left(u t_{+}\right)-\sum_{j^{\prime}=1}^{N} \rho_{j^{\prime}, j} \partial_{x} \varphi_{j^{\prime}}\left(u t_{-}\right)\right] \\
& j_{\mathrm{th}, j}(x, t)=-\left[u \partial_{x} \varphi_{j}\left(u t_{+}\right)\right]^{2}+\left[u \sum_{j^{\prime}=1}^{N} \rho_{j^{\prime}, j} \partial_{x} \varphi_{j^{\prime}}\left(u t_{-}\right)\right]^{2}(21,)
\end{aligned}
$$

where $t_{ \pm}=t \pm x / u$. The effect of the potential bias can be reabsorbed in a shift of the fields

$$
\partial_{x} \bar{\varphi}_{j}\left(t_{ \pm}\right)=\partial_{x} \varphi_{j}\left(t_{ \pm}\right) \pm \frac{e}{2 u} \sqrt{\frac{g}{\pi}} V_{j} .
$$

Switching to the shifted fields in Eq.(22), it is now straightforward to implement the formalism of Appendix $\mathrm{B}$, pertinently generalized to an $N$ QW junction, to compute the average values of the current operators. Retaining only linear contributions in the applied biases, we eventually obtain the electric and thermal conductance tensors,

$$
\begin{aligned}
G_{j, j^{\prime}} & =\frac{e^{2} g}{2 \pi}\left\{\rho_{j, j^{\prime}}-\delta_{j, j^{\prime}}\right\} \\
K_{j, j^{\prime}} & =\frac{\pi k_{B}^{2} T}{6}\left\{\rho_{j, j^{\prime}}^{2}-\delta_{j, j^{\prime}}\right\} .
\end{aligned}
$$

In Eqs. $(23,24)$ we denote with $T$ the equilibrium, reference temperature of the reservoirs and, by definition, we assume that the currents exiting the central island always have positive sign. Similar equations have been derived in Ref.[74] in the framework of a Luttinger liquid in a nonequilibrium steady state. The orthogonality of $\rho$ readily implies the Kirchhoff law for the thermal conductance tensor, $\sum_{j=1}^{N} K_{j, j^{\prime}}=\sum_{j^{\prime}=1}^{N} K_{j, j^{\prime}}=0$.

Concerning the results in Eqs.(23) and (24), it is worth stressing that, throughout the paper, we always assume that particle-hole symmetry holds at equilibrium, which implies that the Seebeck and Peltier coefficients vanish. Formally, this can be traced back to the $\mathbb{Z}_{2}$ symmetry of the bosonic Hamiltonian Eq.(19), for $V_{j}=0$. More generally, in the context of a Tomonaga-Luttinger liquid, particle-hole symmetry breaking may either be determined by, e.g., bulk cubic (or higher-order) interactions arising from nonlinear terms in the fermion dispersion relations, or by local, Sine-Gordon like interactions [37]. In the former case, symmetry breaking operators are typically infrared irrelevant and can be safely neglected throughout our derivation . The latter case takes place for energy-dependent (bare) boundary interaction strengths, a situation not considered in this work.

Taking the ratio between the thermal conductance and the electric conductance across any two leads, one has the Lorenz ratio

$$
\mathcal{L}_{j, j^{\prime}}=\frac{L_{0}}{g}\left(\rho_{j, j^{\prime}}+\delta_{j, j^{\prime}}\right)
$$

In writing Eq.(25), it is implied that $G_{j, j^{\prime}} \neq 0$. Instead, when $G_{j, j^{\prime}}=0$ (and consequently $K_{j, j^{\prime}}=0$ ), as it happens, for instance, at the disconnected fixed point of a junction of $N$ wires discussed in the following, the ratio has to be computed using the finite- $T$ corrections to the conductances within the framework of Appendix E.

From Eq.(25) we identify the two factors that renormalize the Lorenz ratio: a contribution stemming from the interaction in the QWs, encoded in the Luttinger parameter $g$ [13], and a term determined by the tensor structure dictated by the splitting matrix. The former contribution is washed out when the junction is connected to Fermi liquid reservoirs [2, 43-45] (see Appendix $\mathrm{C}$ for an extensive discussion about this point), while the latter term can lead to a violation of the WFL even in the absence of interactions in the QWs. As we are interested in violations of the WFL stemming from the dynamics at the junction, in all the examples that we discuss in the following we attempt to disentangle the two effects and focus onto the contribution arising from the splitting matrix corresponding to a given fixed point.

\section{B. The Wiedemann-Franz law in the $N=3$-junction.}

We now perform a comprehensive analysis of the (violation of) the WFL at the fixed points of a $N=3$ junction of QWs. To do so, we next review the general parametrization of the $\rho$ matrices describing conformal boundary conditions in an $N=3$ junction in bosonic coordinates. Specifically, we first resort to a purely algebraic classification, without addressing the issue of the stability of a specific fixed point (FP) [2, 5, 72, and 73]. At a second stage, we discuss the phase diagram and the FPs that describe the low-temperature physics of specific systems as particular cases of the general results. It is also important to point out that some FPs of the $N=3$ junction, such as the M-fixed point of [2], cannot be described in terms of simple conformal boundary conditions, in bosonic coordinates. However, they admit (in the presence of Fermi liquid reservoirs) a description in terms of a fermionic scattering matrix [7], which, as discussed in section II, implies that the WFL is automatically satisfied.

Let us first assume total charge conservation, i.e., that the electric current in Eq.(21) satisfies the Kirchhoff's law at the junction, as well as the invariance under the $\mathbb{Z}_{3}$ transformation exchanging the leads with each other. Requiring that $\rho$ is orthogonal, as it must be in order to preserve the canonical commutation relations between the bosonic fields, the splitting matrix depends only on the Luttinger parameter $g$ and a real parameter $\vartheta[2]$ as

$$
\rho(\vartheta)=\left(\begin{array}{lll}
a(\vartheta) & b(\vartheta) & c(\vartheta) \\
c(\vartheta) & a(\vartheta) & b(\vartheta) \\
b(\vartheta) & c(\vartheta) & a(\vartheta)
\end{array}\right)
$$


with $-\pi<\vartheta \leq \pi$ and

$$
\begin{aligned}
& a(\vartheta)=\frac{3 g^{2}-1+\left(3 g^{2}+1\right) \cos (\vartheta)}{3\left[1+g^{2}+\left(g^{2}-1\right) \cos (\vartheta)\right]} \\
& b(\vartheta)=\frac{2[1-\cos (\vartheta)+\sqrt{3} g \sin (\vartheta)]}{3\left[1+g^{2}+\left(g^{2}-1\right) \cos (\vartheta)\right]} \\
& c(\vartheta)=\frac{2[1-\cos (\vartheta)-\sqrt{3} g \sin (\vartheta)]}{3\left[1+g^{2}+\left(g^{2}-1\right) \cos (\vartheta)\right]} .
\end{aligned}
$$

Plugging Eqs.(26) and (27) into Eqs.(23) and (24), we eventually obtain the CCT, given by [2]

$$
\mathbb{G}=\frac{e^{2}\left[(\mathbf{1}-3 \mathbb{I}) t_{\vartheta}^{2}+\sqrt{3} g \hat{\epsilon} t_{\vartheta}\right]}{3 \pi\left(g^{2}+t_{\vartheta}^{2}\right)},
$$

and the $\mathrm{HCT}$ given by

$$
\mathbb{K}=-\frac{2 \pi k_{B}^{2} T t_{\vartheta}^{2}}{27\left(g^{2}+t_{\vartheta}^{2}\right)^{2}}\left[\left(3 g^{2}-t_{\vartheta}^{2}\right)(\mathbf{1}-3 \mathbb{I})+2 g \sqrt{3} \hat{\epsilon} t_{\vartheta}\right] .
$$

In Eqs. (28) and (29), the various tensors are defined so that $\mathbf{1}_{i, j}=1, \mathbb{I}_{i, j}=\delta_{i, j}$, and $\hat{\epsilon}_{j, k}=\sum_{l} \epsilon_{j k l}$. Also, we have set $t_{\vartheta}=\tan \frac{\vartheta}{2}$. Taking the ratio between the entries of the conductance tensors with the same pair of indices, we obtain the Lorenz ratio

$$
\mathcal{L}_{j, j^{\prime}}=2 \frac{3 g^{2} \delta_{j, j^{\prime}}+t_{\vartheta}^{2}+\sqrt{3} g t_{\vartheta} \epsilon_{j, j^{\prime}}}{3\left(g^{2}+t_{\vartheta}^{2}\right)} L_{0} .
$$

Our derivation of Eqs. (28) and (29) relies on the existence of the scale invariant matrix $\rho$, characterizing a FP in the phase diagram of the system. Nevertheless, due to the symmetry of the $\rho$ matrix, a generalization of Eqs. (28) and (29) is expected to hold even outside of the FPs, provided that Kirchhoff law for the thermal and for the charge currents is valid and that the boundary interaction Hamiltonian is symmetric under swapping any two leads with each other and exchanging $\vartheta$ with $2 \pi-\vartheta$. In this case, we expect $G_{j, j^{\prime}}$ and $K_{j, j^{\prime}}$ to take the general expression $\mathcal{A}(g, \vartheta, D)\left(1-3 \delta_{j, j^{\prime}}\right)+\mathcal{B}(g, \vartheta, D) \hat{\epsilon}_{j, j^{\prime}}$, with $\mathcal{A}, \mathcal{B}$ being functions of $g, \vartheta$ and of a running dimensionful energy scale $D$ (which in the following we identify with $\left.k_{B} T\right)$.

Admitting explicit breaking of $\mathbb{Z}_{3}$-symmetry, while still requiring charge conservation allows another class of splitting matrices [73 and 75], distinct from the one in Eq. (26), (27). In this case, $\rho_{B}$ does not depend on $g$, as it can be readily checked using the formalism of Appendix $\mathrm{C}$, and its general form is

$$
\rho_{B}=\left(\begin{array}{lll}
\hat{b}(\vartheta) & \hat{a}(\vartheta) & \hat{c}(\vartheta) \\
\hat{a}(\vartheta) & \hat{c}(\vartheta) & \hat{b}(\vartheta) \\
\hat{c}(\vartheta) & \hat{b}(\vartheta) & \hat{a}(\vartheta)
\end{array}\right),
$$

with $\hat{a}(\vartheta), \hat{b}(\vartheta), \hat{c}(\vartheta)$ obtained from $a(\vartheta), b(\vartheta), c(\vartheta)$, respectively, in Eq.(27) by setting $g=1$ [76].

The conductance tensors are directly obtained from Eqs.(29) and (30) by means of the replacement $j^{\prime} \rightarrow 3-j^{\prime}$. Accordingly, we now obtain for the Lorenz ratio

$$
\mathcal{L}_{j, j^{\prime}}=\frac{1}{3}-\delta_{j, j^{\prime}}+\frac{2}{3} \cos \left[\vartheta+\frac{2 \pi\left(j+j^{\prime}\right)}{3}\right] .
$$

An alternative situation of physical interest is the one in which a "dual" Kirchhoff law holds, in that the total charge entering/exiting the junction is equal to zero [77]. Physically, this corresponds to having only Andreev-like scattering processes at the central island (regardless of whether they are single-, or multi-particle), that is, any incoming charge from a lead exits toward either the same or any other lead, as the same charge with opposite sign. By swapping the current and the charge operators with each other (this is equivalent to changing the sign of the chiral $\varphi_{L, j}$ fields, while leaving the one of the $\varphi_{R, j}$ fields unchanged). one obtains the corresponding splitting matrices [78]

$$
\rho_{A}(\vartheta)=-\rho(\vartheta)
$$

with $\rho$ in Eq.(26), with a similar relation holding for $\rho_{B}$. The charge conductance takes the form

$$
G_{j, j^{\prime}}=-\frac{e^{2}}{\pi} \frac{3 g^{2} \delta_{j, j^{\prime}}+t_{\vartheta}^{2}+\sqrt{3} g t_{\vartheta} \epsilon_{j, j^{\prime}}}{3\left(g^{2}+t_{\vartheta}^{2}\right)},
$$

while the heat conductance is still given by Eq.(30). Finally, the Lorenz ratio is given by

$$
\mathcal{L}_{j, j^{\prime}}=-\frac{2\left[\left(1-3 \delta_{j, j^{\prime}}\right) t_{\vartheta}^{2}+\sqrt{3} g \epsilon_{j, j^{\prime}} t_{\vartheta}\right]}{3 \pi\left(g^{2}+t_{\vartheta}^{2}\right)} L_{0} .
$$

For $\vartheta=0$, Eqs. (34) and (35) describe the $D^{3} \mathrm{FP}$ (see Section IV D below).

\section{FIXED POINTS OF JUNCTIONS OF $N$ INTERACTING QUANTUM WIRES}

We now apply the formulas of Section III to compute the CCT and the HCT at several fixed points of a junction of QWs, characterized by conformal boundary conditions such as the ones in Eq.(16). We work with a junction with a generic number $N>2$ of leads [15, 72, 73, and 75], whereas we eventually address specific examples with $N=3[2,6,54,78-82]$. Assuming over-all charge conservation at the junction, generalizing the construction of [2] to the $N$ QW junction, we introduce the center-ofmass $\Phi$ and the relative fields $\xi_{a}(x)(a=1, \ldots, N-1)$ [64]

$$
\left(\begin{array}{c}
\Phi(x) \\
\xi_{1}(x) \\
\vdots \\
\xi_{N-1}(x)
\end{array}\right)=\mathbf{M}_{N} \cdot\left(\begin{array}{c}
\phi_{1}(x) \\
\phi_{2}(x) \\
\vdots \\
\phi_{N}(x)
\end{array}\right)
$$


with the orthogonal matrix

$$
\mathbf{M}_{N}=\left(\begin{array}{cccc}
\frac{1}{\sqrt{N}} & \frac{1}{\sqrt{N}} & \ldots & \frac{1}{\sqrt{N}} \\
\frac{1}{\sqrt{2}} & -\frac{1}{\sqrt{2}} & \ldots & 0 \\
\ldots & \ldots & \ldots & \ldots \\
\frac{1}{\sqrt{N(N-1)}} & \frac{1}{\sqrt{N(N-1)}} & \ldots & -\frac{N-1}{\sqrt{N(N-1)}}
\end{array}\right)
$$

(and similar ones for the $\theta_{j}$ fields). Charge conservation at the junction implies that $\partial_{x} \Phi(0)=0$ [2]. At the disconnected fixed point (DFP), at which all the QWs are disconnected from each other, also the $\left\{\xi_{a}(x)\right\}_{a}$ obey Neumann boundary conditions, which is equivalent to Eqs.(38) below.

A simple way for constructing "nontrivial" FPs with alternative conformal boundary conditions is to trade the boundary conditions in one or more combinations of fields from Neumann to Dirichlet. Pertinently imposing Dirichlet boundary conditions in the relative channels, it is possible to construct FPs characterized by multiparticle scattering processes at the central island. Following the discussion of Section II, we expect these FPs to be good candidates to host a violation of the WFL.

\section{A. The disconnected junction}

The DFP describes disconnected QWs, which is accounted for by imposing open boundary conditions on the system

$$
\rho_{j, j^{\prime}}=\delta_{j, j^{\prime}},
$$

which corresponds to setting $\vartheta=0$ in the right-hand side of Eq.(27). Accordingly, $G_{j, j^{\prime}}=K_{j, j^{\prime}}=0$ for all pairs of indices. While the result at the fixed point is in itself trivial, we employ the conditions in Eq.(38) to write boundary perturbations to the DFP in Section V.

\section{B. The chiral fixed points}

In the noninteracting, $g=1$ limit, we characterize the chiral FPs $\chi^{ \pm}$by the boundary conditions

$$
\varphi_{R, j}(0)=\varphi_{L, j \pm 1}(0) \quad, \quad(j+N \equiv j) \quad .
$$

The corresponding splitting matrix is $\rho_{j, j^{\prime}}=\delta_{j, j^{\prime} \pm 1}$. Physically, this corresponds to perfect transmission of a particle entering from lead $j$ into lead $j \pm 1$, and to zero transmission amplitude into any other lead. For $g \neq 1$, the splitting matrix is constructed using the formulas of Appendix C. For $N=3$, one obtains [2]

$$
\rho_{\chi_{ \pm}}=\frac{2}{3+g^{2}}\left(\begin{array}{ccc}
-\frac{1-g^{2}}{2} & 1 \pm g & 1 \mp g \\
1 \mp g & -\frac{1-g^{2}}{2} & 1 \pm g \\
1 \pm g & 1 \mp g & -\frac{1-g^{2}}{2}
\end{array}\right)
$$

Eqs.(23) and (24) then yield

$$
\begin{aligned}
G_{j, j^{\prime}}^{\chi_{ \pm}} & =\frac{e^{2} g}{\pi\left(3+g^{2}\right)}\left[1-3 \delta_{j, j^{\prime}} \pm g \epsilon_{j, j^{\prime}}\right], \\
K_{j, j^{\prime}}^{\chi_{ \pm}} & =\frac{2 \pi k_{B}^{2} T}{3\left(3+g^{2}\right)^{2}}\left[\left(1+g^{2}\right)\left(1-3 \delta_{j, j^{\prime}}\right) \pm 2 g \epsilon_{j, j^{\prime}}\right]
\end{aligned}
$$

By inspection, the results in Eqs.(41) and (42) imply a violation of the WFL for $g \neq 1$, encoded in the (renormalized) Lorenz ratio

$$
\mathcal{L}_{j, j^{\prime}}=2 \frac{1+g^{2} \delta_{j, j^{\prime}} \pm g \epsilon_{j, j^{\prime}}}{3+g^{2}} L_{0}
$$

Nevertheless the violation is only due to the interaction in the leads and, as discussed in Appendix C, it disappears when the junction is connected to Fermi liquid reservoirs. In this case $\hat{\rho}_{\chi_{ \pm}}=(\mathbf{1} \pm \hat{\epsilon}-\mathbb{I}) / 2$ and the conductance tensors are directly obtained from Eqs.(40) and (41) by setting $g=1$. As stated above, our conclusion applies only provided that the electric conductance tensor component is different from zero. The above discussion clearly applies to the $\chi^{ \pm}$FPs of a generic junction, with any number of leads $N$ and straightforwardly generalizes to any splitting matrix which represents a permutation, i.e., a matrix which has all vanishing entries, except for one off-diagonal entry in each row and in each column. This is the only situation in which the boundary conditions can be equivalently formulated in fermionic variables, $a_{j^{\prime}, j}=r_{j^{\prime}, j}=c_{j^{\prime}, j}=0, \forall j^{\prime}, j=1, \ldots, N$, and $t_{j^{\prime}, j}=\delta_{j^{\prime} \pm 1, j}$ for the $\chi^{ \pm}$FPs. Apparently, in this case the dynamics is described by single-particle scattering processes only.

For $N=3$, the $\chi^{ \pm}$FPs are stable for $1<g<3$ [2]. At variance, for $N \geq 4$ the $\chi^{ \pm}$FPs are unstable for any value of $g$.

\section{The $D^{N-1}$ fixed point}

We consider the FP recovered by imposing Dirichlet boundary conditions onto all the relative fields in Eq.(36), which we generically dub $D^{N-1}$. In the $N=3$ junction, two different types of FPs emerge, denoted by $D_{P}$ and $D_{N}$ in [2]: they share the same splitting matrix, but differ by their operator content and have therefore a different range of stability in the system parameters. Accordingly, while they have the same FP conductance tensors, the finite-temperature corrections will be different, as they scale with a power law of the temperature, which depends on the dimension of the leading irrelevant operator.

The corresponding FP splitting matrix is given by [64 and 65]

$$
\rho_{D^{N-1}}=\frac{2}{N} \mathbf{1}-\mathbb{I} .
$$

Computing the charge and heat conductance tensors with 
the formalism of Section III, we obtain

$$
\mathbb{G}=\frac{e^{2} g}{\pi}\left(\frac{1}{N} \mathbf{1}-\mathbb{I}\right) \quad, \quad \mathbb{K}=\frac{2 \pi k_{B}^{2} T}{3 N}\left(\frac{1}{N} \mathbf{1}-\mathbb{I}\right) .
$$

Eq.(45) is obtained from Eqs.(28) and (29) by setting $\vartheta=\pi$. From Eq.(45) we directly read

$$
\mathcal{L}_{j, j^{\prime}}=\frac{2}{N} \frac{\pi^{2} k_{B}^{2}}{3 g e^{2}}
$$

In Eq.(46) we identify with the factor $g^{-1}$ the contribution merely stemming from the bulk interaction in the QWs (which is washed out once the junction is connected to Fermi liquid reservoirs, see Appendix C for details) and the factor $\frac{2}{N}$ due to the multi-particle scattering processes at the central island. As the renormalization of the Lorenz ratio is present even when the junction is connected to external Fermi liquid leads, we conclude that the WFL breaks down at the $D^{N-1} \mathrm{FP}$, which highlights that the dynamics cannot be described within the singleparticle framework of Section II. In fact, it is directly related to the onset of zero-temperature multi-particle scattering processes at the central island, consistently with the results of [15], where a similar phenomenon was studied at a resonant, multi-lead quantum point contact. Given the $\rho$-matrix in Eq.(44), we may readily identify a scattering process in which $N$ particles are injected into the central island from, e.g., lead $j$. The incoming particles are symmetrically transmitted into the remaining leads as $N-1$ pairs, each of charge $2 e$. As charge is conserved, $N-2$ holes are Andreev backscattered into lead $j$. Alternatively, we may consider a "dual" process in which, e.g., two particles (total charge $2 e$ ) are injected from each lead $j^{\prime}$, with $j^{\prime} \neq j$, and a mix of normal transmission and crossed Andreev reflection yields $N$ particles and $N-2$ holes exiting the central island from lead $j$. In Fig. 3 we draw a sketch of the two processes. Alternatively, we may borrow the second point of view of [15] by considering a single-particle "in" state that, consistently with the picture of Fig.3, gives rise to "out" states in the other leads whose charge is, in general, no longer a multiple of the unit charge $(2 e / N$ in our specific model). This charge fractionalization under scattering at the central island marks an apparent breakdown of the Fermi liquid picture and works as a pictorial explanation of the breakdown of the WFL.

We expect that a pertinent renormalization of the Lorenz ratio also takes place, for $N \geq 4$, at reduced symmetry FPs, such as the $D^{N-2} \mathrm{FP}$, where Dirichlet boundary conditions are imposed to only $N-2$ of the relative fields, while the remaining one obeying Neumann boundary conditions, together with the center-of-mass field.

\section{The $D^{N}$ fixed point}

Compared to the $D^{N-1} \mathrm{FP}$, the $D^{N} \mathrm{FP}$ is characterized by the center-of-mass field $\Phi$ defined in Eq.(36)

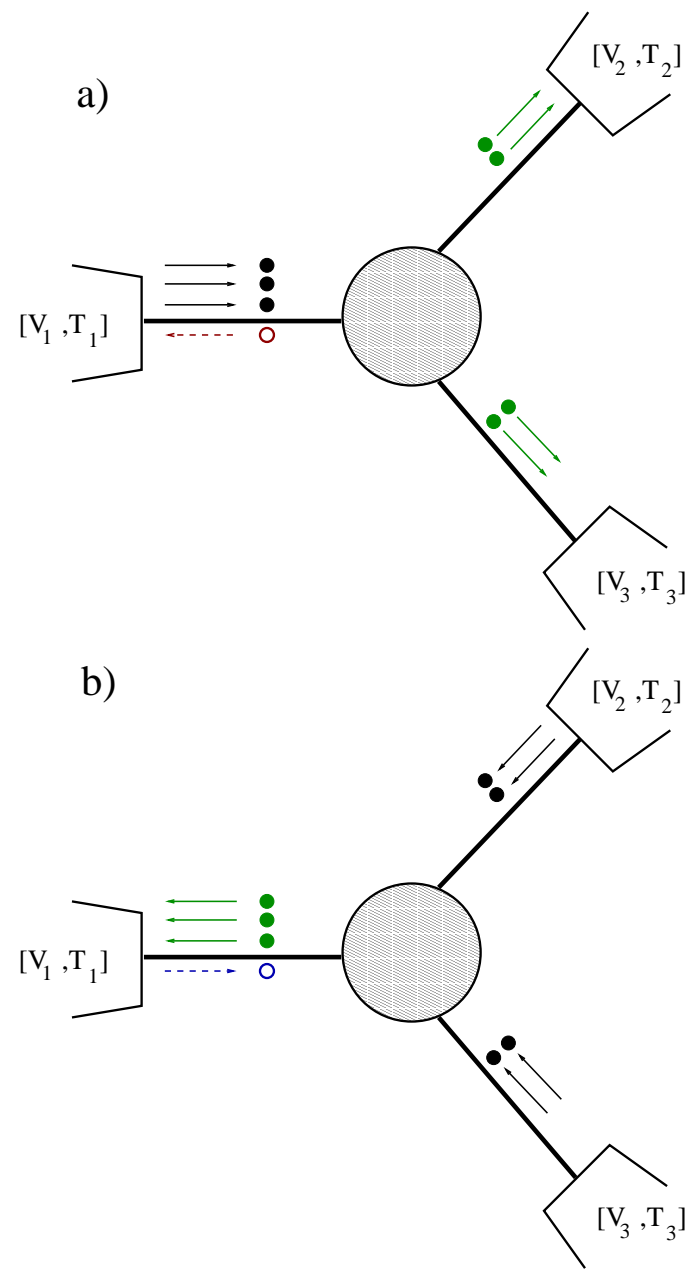

Figure 3. Sketch of two multiparticle scattering processes taking place at the $D^{2} \mathrm{FP}$ of the $N=3$ junction (for $g=1$ ). Specifically,

a): three particles (black full dots) are injected into the central island from lead 1. Two pairs of particles are symmetrically transmitted into leads 2 and 3 (green full dots) while, consistently with the total charge conservation, a hole is Andreev backscattered into lead 1 (red open dot).

b): Two particles are injected from leads 2 and 3 (black full dots) and a mix of normal transmission and crossed Andreev reflection yields an outgoing, multiparticle state within lead 1, consisting of 3 particles (green full dots) and one hole (blue open dot).

satisfying Dirichlet, instead of Neumann, boundary conditions. Correspondingly, the charge conservation breaks down and the WFL can be violated, even though the corresponding FP can be fully described in terms of singleparticle scattering processes only. The $D^{N} \mathrm{FP}$ is dual (in the sense of the charge-current duality [78]) of the DFP, thus, the splitting matrix is given by $\rho=-\mathbb{I}$ and describes perfect Andreev reflection in each lead [64 and 65]. In the language of Section II, $A_{j, j}=1 \forall j$, all the other scattering coefficients being zero. This implies $G_{j, j^{\prime}}=\frac{e^{2}}{\pi} \delta_{j, j^{\prime}}$ and $K_{j, j^{\prime}}=0, \forall j, j^{\prime}=1, \ldots, N$. 
In fact, this is just what happens, at low enough energies, in a single wire coupled to a topological superconductor [31, 83, and 84]. As a result, Andreev reflection becomes a resonant process at the FP, with no room left for normal, single-particle backscattering. In general, when considering the heat conductance through a normal metal-superconductor interface, we find that it strongly depends on the mechanism of electron transfer across the interface. Specifically, when single-particle transfer dominates and, accordingly, the backscattering on the normal side of the interface is mostly normal, electronic states on the normal side at energies above the superconducting gap are depleted, resulting in an effective cooling mechanism of the metal. At variance, when the coherent two-electron tunneling becomes the dominant mechanism for charge transfer across the junction (corresponding to the onset of Andreev reflection on the normal side), the heat flow is strongly suppressed, due to the fact that now electrons with all energies, including those inside the energy gap, are removed from the normal metal [55]. Accordingly, we expect that the full suppression of normal backscattering versus Andreev reflection implies a full suppression of the heat flow through the interface and, in addition, that this conclusion holds regardless of whether the superconducting side is topological.

In conclusion, we may regard the $D^{N} \mathrm{FP}$ as "trivially" violating the WFL. The violation is, indeed, just related to the peculiar subgap physics of the NS-interface. For this reason, in the following we focus on the charge conserving junction with $N=3$.

\section{PHASE DIAGRAM AND TRANSPORT IN CHARGE-CONSERVING $N=3$ JUNCTIONS}

As a specific example of realization of the fixed points described above, we discuss in detail the $N=3$ junction of interacting QWs. Specifically, in the following we focus on two types of boundary interactions: the direct fermion hopping between lead ends in [1 and 2] and the TKM discussed in [27 and 28].

The simplest, nontrivial example is the $N=3$ junction discussed in Refs.[1 and 2], whose generalization to a generic $N(\geq 4)$ is presented in [72]. In such a system, once resorting to the bosonization framework, in terms of the unfolded chiral fields defined in Eq.(17), the boundary interaction at the DFP is given by

$H_{\mathrm{Junc}, N}=-J_{K} \sum_{k<l=1}^{N} \Gamma_{k} \Gamma_{l} e^{i \sqrt{\frac{4 \pi}{g}}\left[\varphi_{k}(0)-\varphi_{l}(0)\right]+i \chi_{k}, l}+$ h.c.,

with $J_{K}$ being the over-all boundary coupling strength and the $\chi_{k, l}$ being phases that may enter $H_{\mathrm{Junc}, N}$ if, e.g., there is a magnetic flux piercing the junction itself [2]. The Hamiltonian in Eq.(47) conserves the total charge, but breaks time reversal invariance for a generic choice of the phases $\chi_{k, l}$. In general, it is a relevant boundary operator as soon as $g>1$.
In the TKM, a superconducting island is present at the junction, hosting low-energy degrees of freedom in the form of MZMs, which are in turn tunnel-coupled to the end of the leads. The superconducting island itself is floating and characterized by a large charging energy $E_{c}$, which ultimately determines the charge conservation at the junction. The boundary Hamiltonian describing such a system in the cotunneling regime is

$$
H_{\mathrm{TK}, 2}=-2 \sum_{k<l=1}^{N} J_{k, l} \cos \left[\sqrt{\frac{4 \pi}{g}}\left(\varphi_{k}(0)-\varphi_{l}(0)\right)+\chi_{k, l}\right] \text {, }
$$

with $J_{k, l} \sim 1 / E_{c}$. No Majorana fields, nor Klein factors appear in Eq.(48), due to the "Majorana-Klein hybridization" [28, 32, and 85], which factors them out of the dynamics. The boundary term can be regarded as a generalization of the Kondo model to the $S O(N)$ symmetry group $\left[27,29\right.$, and 86]. $H_{\mathrm{TK}, 2}$ is relevant for $g>1$ and marginally relevant for $g=1[27,28,32,33,87$, and 88]. Anisotropy in the $J_{k, l}$ are washed out along the renormalization group ( $\mathrm{RG}$ ) trajectories. Accordingly, without any loss of generality, from now on we assume $J_{k, l}=J_{K}$ for every pair of wires.

In a related setting, the superconducting island can be Josephson-coupled to another superconductor, which breaks charge conservation at the junction. In this case, the boundary Hamiltonian is given by [64 and 65]

$$
H_{\mathrm{TK}, 1}=-\sum_{j=1}^{N} \sqrt{2} t_{j} \sin \left[\sqrt{\frac{4 \pi}{g}} \varphi_{j}(0)\right]+H_{\mathrm{TK}, 2}
$$

with $t_{j} \sim E_{J}$ where $E_{J}$ denotes the Josephson energy. The first term always triggers a flow toward a FP at which $\phi_{j}(0)$ is pinned to some nonuniversal value, depending on the specific "bare" values of the boundary interaction strengths [64 and 65]. In the "phase" regime $E_{c} \ll E_{J}$, the low-temperature FP is known as $S O(N)_{1}$ Topological Kondo FP. The first term in Eq.(49) has scaling dimension $(2 g)^{-1}$ and is therefore relevant as soon as $g>\frac{1}{2}$.

We now investigate in detail the various phases with the corresponding transport properties.

\section{A. The disconnected fixed point}

As we discuss in Section IV, at the DFP one finds vanishing conductance tensors. Turning on the boundary interaction, we perturbatively compute the expectation values of the currents by employing the Keldysh approach of Appendix E. As a result, we obtain for the electric and thermal conductance tensors [57]

$$
\begin{aligned}
G_{j, j^{\prime}} & =\frac{6 \pi e^{2} \Gamma^{2}\left(\frac{1}{g}\right) \mathcal{J}^{2}(D)}{\Gamma(2 / g)}\left(\frac{1}{3}-\delta_{j, j^{\prime}}\right), \\
K_{j, j^{\prime}} & =\frac{2 \pi^{3} k_{B}^{2} T \Phi(g) \mathcal{J}^{2}(D) \Gamma^{2}\left(\frac{1}{g}\right)}{\Gamma(2 / g)}\left(\frac{1}{3}-\delta_{j, j^{\prime}}\right),
\end{aligned}
$$


with the dimensionless running coupling $\mathcal{J}(D)=\frac{J_{K}}{D_{0}}\left(\frac{D}{D_{0}}\right)^{-1+\frac{1}{g}}, \quad D=2 \pi k_{B} T$ a scale with the dimension of an energy, and $D_{0}$ a high energy cutoff. The latter is a relevant large energy scale, such as the bandwidth of the conduction band or the charging energy of the floating island in the TKM example in Sec. VB below. As discussed above, due to the system symmetries, we expect that $G_{j, j^{\prime}}=\mathcal{A}_{G}(g, \vartheta, D)\left(1-3 \delta_{j, j^{\prime}}\right)+\mathcal{B}_{G}(g, \vartheta, D) \hat{\epsilon}_{j, j^{\prime}}$ and $K_{j, j^{\prime}}=\mathcal{A}_{K}(g, \vartheta, D)\left(1-3 \delta_{j, j^{\prime}}\right)+\mathcal{B}_{K}(g, \vartheta, D) \hat{\epsilon}_{j, j^{\prime}}$. As it appears from the right-hand side of Eqs. (50) and (51), this is indeed the case. As a consequence, the two conductances have the same tensor structure, hence, the ratio between any pair of nonzero entries is

$$
\mathcal{L}_{j, j^{\prime}}=\Phi(g) L_{0}
$$

with

$$
\begin{aligned}
\Phi(g)= & \frac{3 \Gamma(2 / g)}{g \pi \Gamma^{4}(1 / g)} \int d z d w \frac{z}{\sinh (\pi z)} \\
& \times\left|\Gamma\left(\frac{1}{2 g}+i(z-w)\right) \Gamma\left(\frac{1}{2 g}+i w\right)\right|^{2}
\end{aligned}
$$

As expected, $\Phi(g=1)=1$, which can be shown using the identity (E19) in Appendix E. When $g \neq 1$ but $|g-1| \ll 1$, we may improve the results in Eq.(53) by letting $\mathcal{J}(D)$ flow with the running energy scale $k_{B} T$ according to the appropriate RG equations (see Appendix $\mathrm{F}$ for details). Within the perturbative approach to the TKM, the main effect is the scaling of both $G_{j, k}$ and $K_{j, k}$ with the running coupling. Accordingly, the Lorenz ratio is scale-independent and equal to $L_{0}$ for $g=1$. An important difference between the TKM (48) and the $N=3$ junction (47) emerges for $g=1$, as the boundary interaction in the $N=3$ junction is purely marginal [2]. In this case, Eqs. (50),(51) and (53) provides the leading perturbative (in $J_{K}$ ) contributions to the conductance tensors at a manifold of Fermi liquid fixed point, consistently with the result $\Phi(1)=1$. Conversely, the boundary interaction (48) is marginally relevant for $g=1$ : in this case, we cannot rely on the above results at low temperatures, but we rather need to assess the stable fixed point encoding the $T \rightarrow 0$ behavior of the junction. To do so, we now go through an extensive review of the phase diagram of the $N=3$ junction and of the TKM.

\section{B. Phase diagram of the $N=3$ junction and of the topological Kondo model}

While our derivation allows us to make a sharp prediction on the value of the Lorenz ratio, in order for the effect to be robust in a realistic system, the $D^{N-1} \mathrm{FP}$ has to be an infrared attractive RG fixed point. In junctions of normal QWs, this happens only at rather unphysically large values of the (attractive) interaction strength in the leads [1 and 2]. The $D^{N-1} \mathrm{FP}$ also emerges in the phase diagram of the TKM [27, 28, and 64]: remarkably, it is stable as soon as $g>\frac{N}{2(N-1)}[27$ and 28]. This points toward an intriguing relation between the emergence of MZMs and the detection of a robust violation of the WFL as discussed above, so that the latter effect may be used as an evidence for the presence of MZMs. In order to better spell out this point, we discuss the case $N=3$, for which a complete classification of the FPs and of the corresponding conformal boundary conditions is possible [2 and 89]. We assume for simplicity $\chi_{l, l+1}=\chi / 3$, see Eqs. (47) and (48), and $-\pi<\chi \leq \pi$ throughout this section.

\section{Direct hopping}

The phase diagram of the $N=3$ junction with direct hopping between the leads Eq.(47) has been discussed in detail in [2]. For $g<1$ (repulsive interaction in the leads), the DFP is infrared (IR) stable. In the absence of interaction, $g=1$, the junction has a manifold of marginally equivalent FPs, which can be described in terms of the single-particle $\mathbf{S}$-matrix approach of Section II. For $1<g<3$, the system flows instead outside of the weakly coupled regime. Any $\chi \neq 0, \pm \pi$ breaks timereversal invariance, triggering a nontrivial renormalization toward either one (depending on the sign of $\chi$ ) of the chiral FPs of sec. IV B, which are stable as long as $g<3$. For the sake of our discussion, it is useful to remind that, in the noninteracting limit, the chiral FPs $\chi_{ \pm}$can be described within the single-particle $\mathbf{S}$-matrix formalism as well. When $\chi=0$, the RG flow points instead toward a time-reversal invariant, finite coupling FP, dubbed MFP in [2]. While a full theory of the MFP is still lacking, based on the numerical results of [30 and 54], in the following we argue that the WFL is expected to hold at the MFP as well, once the junction is connected to external reservoirs. For $g=3$ two disconnected FP manifolds emerge, respectively connected to either one of the chiral FPs, separated from each other by the MFP. For $3<g<9$ two IR stable strongly coupled FPs emerge in the phase diagram, the $D_{P} \mathrm{FPs}$, while the $\chi_{ \pm}$FPs disappear. The $D_{P}$ FPs are still separated by a time-reversal invariant FPs $\mathbf{M}^{\prime}$, analogous to the MFP. Finally, for $g>9$ the $\mathbf{M}^{\prime}$ FPs disappear, as well. Along the time-reversal invariant RG trajectories, the junction flows toward the $D_{N}$ FP. The $D_{P}$ and the $D_{N}$ FPs are described by the same $\rho$ matrix, that is, $\rho_{D^{2}}$ in Eq. (44) with $N=3$, but differ in their operator content. The $D_{P}$ FPs are stable for $g>3$, the $D_{N}$ FPs are stable for $g>9[2]$. In order to stabilize these FPs, we need a large value of the Luttinger parameter, corresponding to a very strong attractive bulk interaction, hard to realize in realistic junctions.

In Fig.4, we draw the RG trajectories of the $N=3$ junction within the various range of parameters discussed above. In order to evidence our main conclusions, we highlight as black/green dots the FPs in the phase dia- 


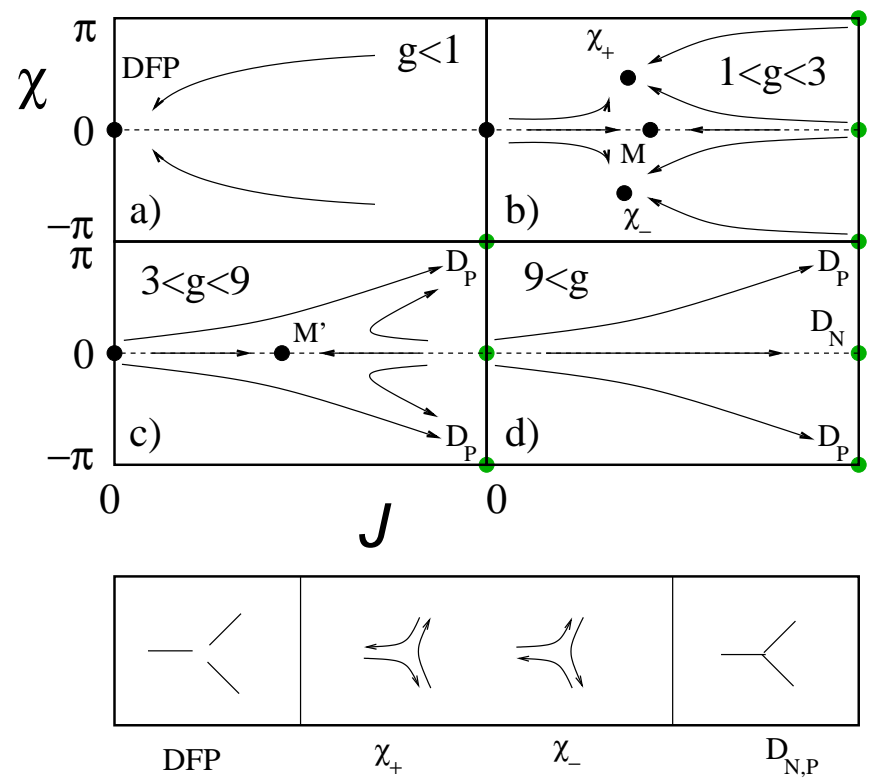

Figure 4. Top: Sketch of the RG trajectories of the $N=3$ junction in the $\mathcal{J}-\chi$ plane within the relevant ranges of values of the Luttinger parameter $g$. We mark with green dots the FPs where a violation of the WFL is expected, also when the junction is connected to Fermi-liquid reservoirs. Specifically, a) $g<1$, only the DFP is stable and the WFL holds;

b) $1<g<3$ : depending on the value of $\chi$ at the reference scale, the system flows toward either the $\mathbf{M}$, or to the $\chi_{ \pm}$ FPs. In any of these FPs the WFL holds;

c) $3<g<9$ : the $D_{P}$ FPs become stable and the WFL breaks down;

d) $9<g$ : both the $D_{P}$ and the $D_{N}$ FPs become stable. In both cases the WFL breaks down.

Bottom: Pictorial sketch of the FPs relevant to our analysis. From left to right: the DFP (all the wires are disconnected from each other), the $\chi_{ \pm}$FPs, and the (fully connected) $D_{N, P}$ FPs.

gram at which the WFL holds/breaks down, when the junction is connected to external leads, as we discuss in Section V C.

\section{The Topological Kondo model}

The RG flow of the TKM has been discussed in $[27$ and 28], as well as in [58 and 90], and the effect of a nonzero $\chi$ has been considered in [25 and 91]. Here, we summarize the corresponding equations in Appendix F. For a small "bare" coupling $\mathcal{J}>0$ at the reference scale $D_{0}$, the boundary Hamiltonian in Eq.(48) is relevant for $g>1$. At the same time, for $\chi \neq \pm \pi$, any nonzero $\chi$ renormalizes to zero. For $g \geq 1$, the system flows towards a large- $\mathcal{J} \mathrm{FP}$, which corresponds to the chargeconserving $D^{2} \mathrm{FP}$. The corresponding splitting matrix has been determined in [64] and is given in Eq.(44) for $N=3$. With this knowledge, we can define the "unfolded", chiral fields

$\tilde{\varphi}_{j}(x)=\left\{\begin{array}{c}\varphi_{L, j}(x), \quad(0 \leq x \leq \ell) \\ \sum_{j^{\prime}=1}^{3}\left[\rho_{D^{2}}\right]_{j^{\prime}, j} \varphi_{R, j^{\prime}}(-x),(-\ell \leq x<0)\end{array}\right.$.

In terms of the fields in Eq.(54), the leading boundary perturbation is given by [58]

$$
\tilde{H}_{\mathrm{TK}, 2}=-2 h \sum_{j=1}^{3} \cos \left[\frac{4 \sqrt{\pi g}}{3}\left(2 \tilde{\varphi}_{j}-\tilde{\varphi}_{j+1}-\tilde{\varphi}_{j-1}\right)\right],
$$

with $\tilde{\varphi} \equiv \varphi_{j}(0)$ and $j+3 \equiv j$. In the formalism of Appendix E, Eq.(55) corresponds to setting

$$
\begin{aligned}
& \alpha_{1,2,3}^{2,3}=\frac{2 \sqrt{g}}{3}(-2,1,1) \\
& \alpha_{1,2,3}^{3,1}=\frac{2 \sqrt{g}}{3}(1,-2,1) \\
& \alpha_{1,2,3}^{1,2}=\frac{2 \sqrt{g}}{3}(1,1,-2) .
\end{aligned}
$$

The operator in Eq.(55) has scaling dimension $\frac{4 g}{3}$. Therefore, the topological Kondo FP for $N=3$ is stable as long as $g>\frac{3}{4}$. Thus, we conclude that the DFP is attractive as long as $g<1$ and $\chi \neq \pm \pi$, while the topological Kondo FP is attractive for $g>\frac{3}{4}$ and $\chi \neq \pm \pi$. At variance, for $g \geq 1$ and $\chi \neq \pm \pi$, the system flows toward the topological Kondo FP described by the splitting matrix $\rho_{D^{2}}(44)$ and with leading perturbation in Eq.(55). From the analysis of Appendix $\mathrm{F}$, we conclude that $\chi$ does not flow along the fixed lines $\chi= \pm \pi$. In this case, by explicit investigation one finds that the leading boundary interaction at the Topological Kondo FP (which we dub $\hat{D}^{2}$ in the following) has scaling dimension $\frac{4 g}{9}[25]$. In Eq.(F7) we provide the explicit formula for the leading boundary perturbation: it has, in fact, scaling dimension $\frac{4 g}{9}$ and is therefore relevant as long as $g \leq \frac{9}{4}$. Accordingly, for $\chi= \pm \pi$, there is a finite window $1<g<\frac{9}{4}$ in which both the DFP and the $D^{2} \mathrm{FP}$ are unstable and there appears a stable, finite coupling fixed point for RG trajectories originating from both the DFP and the $\hat{D}^{2} \mathrm{FP}$ [25]. Analogously to the MFP of [2], no complete theory exists for the intermediate-coupling fixed point and we are so far unable to make a sharp prediction on the corresponding behavior of the HCT and on the possible violation of the WFL.

Also in the regime $g<1$, we recover the RG fixed lines $\chi=0$ and $\chi= \pm \pi$, the former one being attractive, the latter one repulsive. For $\frac{3}{4}<g<1$, both the weakly coupled DFP and the strongly coupled $\hat{D}^{2} \mathrm{FP}$ are stable. This implies the emergence of a repulsive finite-coupling FP in the phase diagram, corresponding to a quantum phase transition between the two of them at $\mathcal{J}=\tilde{\mathcal{J}}_{K}=$ $\frac{g^{-1}-1}{2}, \chi=0[58]$.

Finally, we note that, while there is no reason to exclude $a$ priori the emergence of the $\chi_{ \pm}$FPs in the phase diagram of the TKM, we are not able to recover them as 


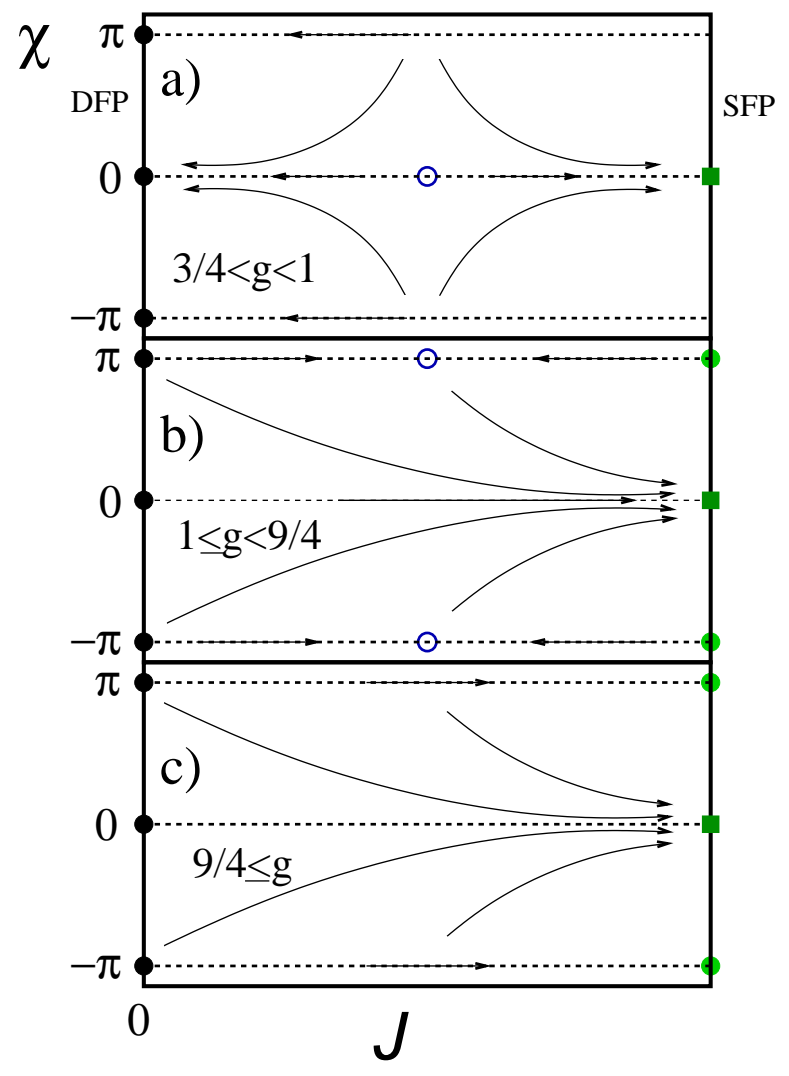

Figure 5. Sketch of the RG trajectories of the TKM in the $\mathcal{J}-\chi$ plane for various ranges of values of the Luttinger parameter $g$. Here SFP denotes any strong-coupling fixed point. The green dots are the FPs where the WFL does not hold for the junction connected to Fermi liquid reservoirs. (a) $\frac{3}{4}<g<1$ : At $\chi \neq \pm \pi$, both the DFP (black dot) and the $D^{2}$ (dark green square) FPs are stable. The running phase $\chi(D)$ (see Appendix $\mathrm{F}$ for details) renormalizes back to the fixed line $\chi=0$. For certain values of the bulk interactions, a repulsive, finite coupling FP appears (open blue dot).

$\mathbf{b}() 1 \leq g<\frac{9}{4}$ : For $\chi\left(D_{0}\right) \neq \pm \pi, \chi(D)$ again flows toward the $\chi=0$ fixed line. $\mathcal{J}(D)$ is renormalized to strong coupling: the $D^{2} \mathrm{FP}$ is stable and, accordingly, the WFL breaks down as $T \rightarrow 0 . \chi= \pm \pi$ are two fixed lines (in $\chi$ ), along which the DFP and the $\hat{D}^{2} \mathrm{FP}$ are repulsive. The actual stable phase corresponds to the finite coupling FP represented by the open blue dot: its actual nature deserves further investigation;

(c) $\frac{9}{4} \leq g$ : Both the $D^{2} \mathrm{FP}$ and the $\hat{D}^{2} \mathrm{FP}$ become stable, thus, the WFL breaks down as $T \rightarrow 0$, regardless of the initial value of $\chi$.

endpoints of RG trajectories fully lying within the $\mathcal{J}-\chi$ plane, differently to what happens in the $N=3$ junction of [2]. Indeed, in order to get access to the time-reversal breaking FPs, one has to introduce an additional, "chiral" boundary interaction, e.g., the analog of the boundary interaction discussed in [92 and 93] at a $Y$-junction of critical Heisenberg chains, which we do not discuss here.

To summarize the discussion about the TKM, in Fig.5, we draw the RG trajectories for the system for $g<1$ in Fig.5(a) and for $g \geq 1$ in Fig.5(b)) and (c).
C. Charge and thermal conductance and the Wiedemann-Franz law in the three-wire junctions

We now review the charge and the thermal conductance at the "nontrivial" FPs of the $N=3$ junction and of the TKM and briefly discuss the scaling properties of the corresponding conductance tensors. We begin with the MFP in the $N=3$-junction: its emergence was originally inferred from the main topology of the phase diagram [1 and 2]. Later on, it was confirmed within a combined use of boundary conformal field theory and numerical density matrix RG approach [30 and 54], eventually showing that the corresponding $\mathrm{CCT}$ for the junction connected to external Fermi liquid reservoirs is given by

$$
\mathbb{G}_{\mathbf{M}}=\frac{e^{2} \gamma}{2 \pi}(\mathbf{1}-3 \mathbb{I})
$$

with $\gamma=\frac{4}{9}$, within numerical error bars [30]. Using the $\mathrm{RG}$ approach in fermionic coordinates, in [7] it was proposed that the conductance in Eq.(57) is determined by the single-particle $\mathbf{S}$-matrix $\mathbf{S}_{M}$, which describes scattering processes at the junction connected to the reservoirs, given by

$$
\mathbf{S}_{M}=\left(\frac{2}{3} \mathbf{1}-\mathbb{I}\right)
$$

Following the derivation of Section II and relating to general arguments based on the scattering matrix description of the junction (see, e.g., [56]), we conclude that the WFL holds at the MFP.

In general, however, when the junction is not connected to Fermi liquid reservoirs, we infer from Eqs.(57) and (58) that we cannot describe the MFP, within the bosonization framework, in terms of an orthogonal splitting matrix. We may instead still define a non orthogonal matrix

$$
\tilde{\rho}=\frac{1}{3}\left(\frac{4}{3} \mathbf{1}-\mathbb{I}\right)
$$

to describe the linear relations between the chiral electric current operators at the junction. The formalism of Appendix C cannot be exploited, as it is based on the possibility of expressing the currents in bosonic language. The non-orthogonality of the matrix in Eq.(59), however, implies that it is not possible to describe the MFP in terms of conformal boundary conditions on the chiral bosonic fields.

In order to capture the main behavior of the junction connected to Fermi liquid reservoirs, we refer to Eqs. (50) and (51). The conductances both receive corrections proportional to $\mathcal{J}\left(D=2 \pi k_{B} T\right)$. On connecting the junction to the reservoirs, there is a crossover in the scaling properties as soon as $\frac{\beta}{2 \pi} \sim \frac{\ell}{\pi u}$. At lower temperatures, we expect the scaling behavior for $g=g_{\text {Res }}=1$ : the boundary interaction becomes, therefore, marginal and can change the $\mathbf{S}$-matrix of the junction. The MFP, as 
well as the $\chi_{ \pm}$FPs, are just specific points over the manifold spanned along the above marginal deformation. Accordingly, they can all be equivalently described in terms of a single-particle $\mathbf{S}$ matrix. At each point of that manifold the WFL holds [56].

At the $D^{2} \mathrm{FPs}$, the WFL is instead violated, with the Lorenz ratio computed in Eq. (46). Employing the formalism of Appendix E, we may write the scaling functions for the conductance tensors, as well as for the renormalization factor of the Lorenz ratio, once we know the leading boundary perturbation allowed by the symmetries of the FP. For the $N=3$ junction, it was shown in [2] that the leading boundary perturbation corresponds to a linear combination of boundary operators with scaling dimension $\Delta_{P}=\frac{g}{3}$ at the $D_{P}$ FPs and $\Delta_{N}=\frac{g}{9}$ at the $D_{N}$ FPs.

From scaling arguments and symmetry considerations, we expect for the conductance tensors in the vicinity of the FPs the general expressions

$$
\begin{aligned}
& \mathbb{G}(T)=\mathbb{G}^{*}-\frac{e^{2} \tilde{h}^{2}\left(2 \pi k_{B} T\right)}{2 \pi}(3 \mathbb{I}-\mathbf{1}) \Phi_{\mathrm{el}}(g) \\
& \mathbb{K}(T)=\mathbb{K}^{*}-\frac{\pi k_{B}^{2} \tilde{h}^{2}\left(2 \pi k_{B} T\right) T}{6}(3 \mathbb{I}-\mathbf{1}) \Phi_{\mathrm{th}}(g),
\end{aligned}
$$

with the dimensionless effective coupling $\tilde{h}(D)=\frac{h}{D_{0}}\left(\frac{D}{D_{0}}\right)^{-1+\Delta_{D_{P}\left(D_{N}\right)}}$ and $\mathbb{G}^{*}, \mathbb{K}^{*}$ the FP conductance tensors in Eqs.(45). Eqs.(60), with $\Phi_{\mathrm{el} / \mathrm{th}}(g)=\Phi_{\mathrm{el} / \mathrm{th} ; D_{P}\left(D_{N}\right)}(g)$, can be readily recovered using the formulas of Appendix E. According to Eqs.(60), we find a corresponding renormalization of the Lorenz ratio given by

$$
\mathcal{L}(T) \approx L_{0}\left\{\frac{2}{3}+\left[\Phi_{\mathrm{th}}(g)-\Phi_{\mathrm{el}}(g)\right] \tilde{h}^{2}\left(2 \pi k_{B} T\right)\right\} .
$$

As expected, when the FP is attractive, the finite- $T$ corrections to the FP conductance tensors, as well as to the Lorenz ratio, scale to zero as $T \rightarrow 0$. When, instead, the FP is unstable against a finite boundary coupling, we see from Eqs. (60) and (61) that the perturbative regime breaks down as soon as $\tilde{h}\left(2 \pi k_{B} T\right) \sim 1$. Considering the case in which the junction is connected to the external reservoirs, we expect that Eqs. (60) and (61) cease to be valid once again at a scale $\left(2 \pi k_{B} T_{c}\right)^{-1} \sim \frac{\ell}{\pi u}$. At lower temperatures, $h$ is traded for the running coupling extracted at the scale $T_{c}$ and with $\Delta_{P}=\frac{1}{3}, \Delta_{N}=\frac{1}{9}$. We summarize the phase diagram for the connected $N=3$ junctions in Fig.6. We see that the system always flows back to the fixed point manifold described in terms of a single-particle $\mathbf{S}$ matrix. Therefore, we always recover the WFL at low enough $T$.

In the RG flow of the TKM, two nontrivial fixed point appear. At the $D F P$, for $N=3$, the leading boundary perturbation is a linear combination of operators with scaling dimension $\Delta_{D}=\frac{4 g}{3}[27,28,58$, and 94]. At the $\hat{D F P}$, only reached along the fixed lines

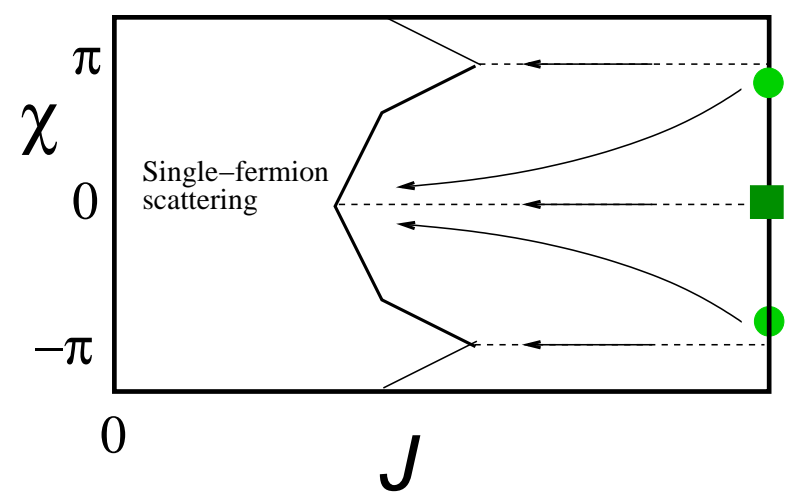

Figure 6. Sketch of the RG trajectories in the $\mathcal{J}-\chi$ plane for the $N=3$ junction connected to Fermi liquid reservoirs. The $D_{P}$ and the $D_{N}$ FPs (light green dots and dark green squares, respectively) are both repulsive. The RG trajectories always flow back toward the fixed manifold each point of which is described in terms of single-fermion scattering processes. Accordingly, the WFL is expected to be preserved as $T \rightarrow 0$, regardless of the initial values of the junction parameters.

$\chi= \pm \pi$, the leading boundary perturbation has scaling dimension $\Delta_{\hat{D}}=\frac{4 g}{9}$ [94 and 95]. In the vicinity of the FPs the conductance tensors have the form (60), with $\tilde{h}=h\left(\frac{\beta}{2 \pi}\right)^{1-\Delta_{D(\hat{D})}}$ and $\Phi_{\mathrm{el} / \mathrm{th} ; D(\hat{D})}(g)$ nonuniversal functions of $g . G(T)$ and $K(T)$ flow to their fixedpoint value with a leading, finite- $T$ correction scaling as $T^{2\left(\Delta_{D}-1\right)}$ and as $T^{2 \Delta_{D}-1}$, respectively [57]. The Lorentz ratio is corrected as in (61), but this time in the connected junction and with $\tilde{h}=h\left(\frac{\beta}{2 \pi}\right)^{-\frac{1}{3}}$. In Fig.7 we summarize the boundary RG flows of the TKM, to be compared to the one in Fig.6. The stable phase is completely different from the one that emerges in the simple $N=3$ connected junction. This is due to the peculiar scattering dynamics at the junction, tightly related to the emergence of the MZMs at the central island and to the hybridization between the MZMs and the Klein factors used in the bosonization of the leads [32 and 85], which in turn washes out the effect of the Klein factors that destabilize the $D^{N-1} \mathrm{FP}$ in a junction of normal wires $[2,25$, and 94]. These considerations eventually lead to the proposal of synoptically looking at the charge and at the thermal transport properties of the junction, as an alternative mean to characterize the MZMs at the island [57].

Before concluding this Section, it is worth stressing that, in the specific context of the Kondo effect, a violation of the WFL has been evidenced as $T$ is of the order of the Kondo temperature $T_{K}[48]$. As we discuss in Appendix F, in our specific case, $T_{K}$ is defined as the temperature scale at which the perturbation theory breaks down and the running couplings become of order 1 [27 and 57]. Our result in Eq. (50) is expected to apply only close to the DFP, for $\mathcal{J}_{K}\left(2 \pi k_{B} T\right) \ll 1$, or, equivalently, $T \gg T_{K}$. Conversely, our results in Eqs. (45) and (60) hold near by the strongly coupled FP [57] 


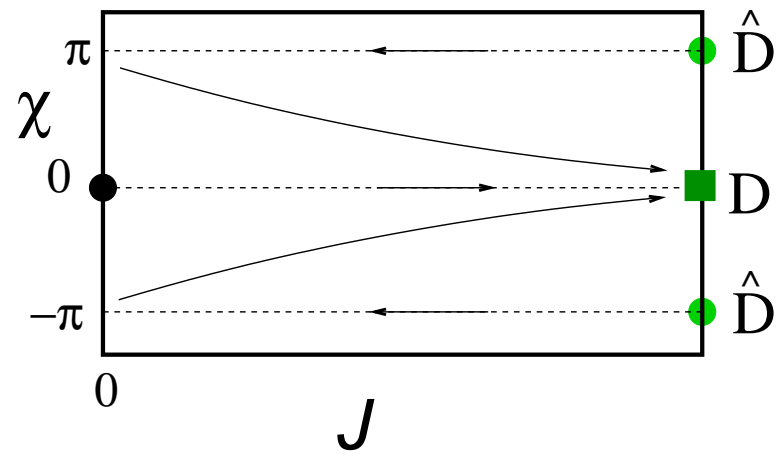

Figure 7. Sketch of the RG trajectories in the $\mathcal{J}-\chi$ plane for the TKM connected to Fermi liquid reservoirs. The $D$ and the $\hat{D}$ FPs (dark green squares and light green dots, respectively) are one attractive, the other(s) repulsive. The RG trajectories flow toward the DFP anywhere, except for $\chi= \pm \pi$. Accordingly, at any $\chi \neq \pm \pi$, we expect the Wiedemann-Franz to break down as $T \rightarrow 0$, regardless of the initial values of the junction parameters.

and are therefore valid for $T \ll T_{K}$. For $g<1$, instead, the perturbative results are also reliable for $T \ll T_{K}$, provided the bare coupling strength is below the critical value given in section VB2. In general, the full scaling curve (as a function of $T / T_{K}$ ) for both the charge and the thermal conductance has been numerically derived in [48], getting two different values for $T_{K}$ from the two scaling curves. Our result in Eqs. (50) and (51) implies that $G(T)$ and $K(T) / T$ scale with the same function of $T$, up to a factor $L_{0}$. In fact, our perturbative derivation possibly misses higher-order contributions in $\mathcal{J}$ to the right-hand side of Eqs. (50) and (51) which, in principle, might render the scaling function associated to $G(T)$ and to $K(T) / T$ no longer different by only a factor $L_{0}$. Yet, while this is likely to affect the numerical estimate(s) of the Kondo temperature, it is not expected to spoil our fixed points (and close to the fixed point) results.

\section{CONCLUSIONS}

In this paper we determined the charge and the thermal conductance tensors at various fixed points in the phase diagram of a junction of $N$ interacting quantum wires. We showed the direct connection between the onset of Andreev reflection and/or crossed Andreev reflection processes and the violation of the Wiedemann-Franz law determined by the corresponding "charge-heat separation" $[38,56$, and 96]. In the specific case in which the total charge is conserved at the junction, we have shown that the breakdown of the Wiedemann-Franz law is directly related to the onset of multiparticle scattering processes and that it is different from the "trivial" breakdown determined by interactions. For $N=3$ wires, we have explicitly computed the Lorenz ratio for a wide class of boundary conditions.

Among the possible mechanisms stabilizing a phase with multiparticle scattering at the central island, we have pointed out the role of the bulk interaction in the leads and explored the consequences of the coupling between isolated Majorana modes at the central island and the low-energy modes in the leads. We have highlighted that, when connecting the junction to external, Fermi liquid reservoirs (as fairly common in transport experiments), the former mechanism is deactivated, while the latter mechanism remains effective. In this paper and in [57], we have explored the direct relation between the breakdown of the Wiedemann-Franz law and the presence of Majorana zero modes in the junction.

The effectiveness of the combined analysis of the charge and heat transport properties of a junction of quantum wires to unveil the relevant physics that sets in at nontrivial fixed points in the phase diagram of the system suggests extensions of our approach to, e.g., junctions of quantum spin chains (where the charge current has to be substituted with a pertinent definition of the spin current) $[20,22,23$, and 86], or to junction of bosonic systems [82], such as cold atom condensates [26]. We plan to go through this topic as a further extension of our work.

Acknowledgements: We thank M. Burrello for the valuable feedback. F.B. and R.E. were funded by the Deutsche Forschungsgemeinschaft (DFG, German Research Foundation) under Germany's Excellence Strategy Cluster of Excellence Matter and Light for Quantum Computing (ML4Q) EXC 2004/1 390534769 and Normalverfahren Projektnummer EG 96-13/1 and under Projektnummer 277101999 - TRR 183 (project B04). A. N. was financially supported by POR Calabria FESR-FSE 2014/2020 - Linea B) Azione 10.5.12, grant no. A.5.1. D.G. acknowledges financial support from Italy's MIUR PRIN project TOP-SPIN (Grant No. PRIN 20177SL7HC).

\section{Appendix A: Lattice model for a junction of $N$ noninteracting quantum wires}

In this Appendix we briefly review the derivation of the $\mathrm{CCT}$ and of the HCT in a lattice model of a junction of $N$ noninteracting quantum wires. In particular, we assume that, as the temperature $T \rightarrow 0$, only single-particle scattering processes take place at the central island: we allege a particle/hole entering from wire $j$ to undergo a normal reflection within the same wire as a backscattered particle/hole, a normal transmission to wire $j^{\prime}(\neq j)$ as a particle/hole, an Andreev reflection within the same wire as a hole/particle, or a crossed Andreev reflection to wire $j^{\prime}(\neq j)$ as a hole/particle. To account for all these processes we employ a pertinently adapted lattice version of the two-component Nambu spinor formalism of [97]. Regarding each lead as an $\ell$-site lattice with hopping strength $J$ and chemical potential $\mu$, we write the 
lattice lead Hamiltonian as $H_{\text {Lat }, 0}=\sum_{j=1}^{N} H_{\text {Lat }, 0, j}$, with

$$
H_{\mathrm{Lat}, 0, j}=-J \sum_{r=1}^{\ell-1}\left[c_{r, j}^{\dagger} c_{r+1, j}+c_{r+1, j}^{\dagger} c_{r, j}\right]-\mu \sum_{r=1}^{\ell} c_{j, r}^{\dagger} c_{j, r},
$$

with $c_{r, j}, c_{r, j}^{\dagger}$ being single-fermion annihilation/creation operators at site $r$ of lead $j$. Within our formalism, we write the wavefunction for an incoming particle from wire $j$, evaluated at site $r$ of wire $j^{\prime}$ as

$$
\begin{gathered}
{\left[\begin{array}{c}
u_{r ; \epsilon ; j^{\prime}}^{(j, p)} \\
v_{r ; \epsilon ; j^{\prime}}^{(j, p)}
\end{array}\right]=\delta_{j, j^{\prime}} \mathcal{N}_{p}\left[\begin{array}{c}
e^{-i k_{p}(r-1)}+r_{j^{\prime}, j^{\prime}}(\epsilon) e^{i k_{p}(r-1)} \\
a_{j^{\prime}, j^{\prime}}(\epsilon) e^{-i k_{h}(r-1)}
\end{array}\right]} \\
+\left[1-\delta_{j, j^{\prime}}\right] \mathcal{N}_{p}\left[\begin{array}{c}
t_{j, j^{\prime}}(\epsilon) e^{i k_{p}(r-1)} \\
c_{j, j^{\prime}}(\epsilon) e^{-i k_{h}(r-1)}
\end{array}\right], \quad(\mathrm{A} 2)
\end{gathered}
$$

with $r_{j, j}(\epsilon), a_{j, j}(\epsilon), t_{j, j^{\prime}}(\epsilon), c_{j, j^{\prime}}(\epsilon)$ respectively corresponding to the normal reflection amplitude within wire $j$, to the Andreev reflection amplitude within wire $j$, to the normal transmission amplitude from wire $j$ to wire $j^{\prime}$, and to the crossed Andreev reflection amplitude from wire $j$ to wire $j^{\prime}$. Also, we write the similar wavefunction for an incoming hole from lead $j$ as

$$
\begin{gathered}
{\left[\begin{array}{c}
u_{r ; \epsilon \in j^{\prime}}^{(j, h)} \\
v_{r ; \epsilon ; j^{\prime}}^{\left(j, h h^{\prime}\right.}
\end{array}\right]=\delta_{j, j^{\prime}} \mathcal{N}_{h}\left[\begin{array}{c}
\tilde{a}_{j^{\prime}, j^{\prime}}(\epsilon) e^{i k_{p}(r-1)} \\
e^{i k_{h}(r-1)}+\tilde{r}_{j^{\prime}, j^{\prime}}(\epsilon) e^{-i k_{h}(r-1)}
\end{array}\right]} \\
\left.+\left[1-\delta_{j^{\prime}, j}\right] \mathcal{N}_{h}\left[\begin{array}{c}
\tilde{c}_{j, j^{\prime}}(\epsilon) e^{i k_{p}(r-1)} \\
\tilde{t}_{j, j^{\prime}}(\epsilon) e^{-i k_{h}(r-1)}
\end{array}\right], \quad, \quad \text { (A3 }\right)
\end{gathered}
$$

with the amplitudes $\tilde{r}_{j, j}(\epsilon), \tilde{a}_{j, j}(\epsilon), \tilde{t}_{j, j^{\prime}}(\epsilon)$ and $\tilde{c}_{j, j^{\prime}}(\epsilon)$ having the same meaning as those in Eq.(A2). The scalars $\mathcal{N}_{p, h}$ are normalization constants and the momenta $k_{p, h}$ are defined as a function of the energy by the relations $\epsilon=-2 J \cos \left(k_{p}\right)-\mu=2 J \cos \left(k_{h}\right)+\mu$. Denoting with $\left\{c_{\epsilon, p, j}, c_{\epsilon, h, j}\right\}$ the single-fermion annihilation operators in the state corresponding to a particle/hole entering the central island from lead $j$ at energy $\epsilon$, we write the real-space, lattice single-fermion operators as

$$
c_{r, j}=\sum_{\epsilon>0} \sum_{j^{\prime}=1}^{N} \sum_{u=p, h}\left\{u_{r ; \epsilon ; j}^{\left(j^{\prime}, u\right)} c_{\epsilon, u, j^{\prime}}+\left[v_{r ; \epsilon ; j}^{\left(j^{\prime}, u\right)}\right]^{*} c_{\epsilon, u, j^{\prime}}^{\dagger}\right\} .
$$

Expanding $c_{r, j}$ by retaining only the low-energy, longwavelength excitations around the Fermi momenta $\pm k_{F}= \pm \arccos \left(-\frac{\mu}{2 J}\right)$, we can recast it in the form

$$
c_{r, j} \approx e^{i k_{F} r} \psi_{R, j}\left(x_{r}\right)+e^{-i k_{F} r} \psi_{L, j}\left(x_{r}\right),
$$

with $x_{r}=a r$ and $a$ being the lattice step (which we set to unity elsewhere in the paper). $\psi_{R, j}(x), \psi_{L, j}(x)$ are the chiral fields that we used throughout the derivation of Section II, where we resorted to the continuum variable framework. Here, instead, we keep using the lattice formalism, in which the current operators in lead $j$ are given by

$$
\begin{aligned}
& j_{\mathrm{el}, r, j}=-i e J c_{r, j}^{\dagger} c_{r+1, j}+\text { h.c. } \\
& j_{\mathrm{th}, r, j}=i J^{2} c_{r-1, j}^{\dagger} c_{r+1, j}+\frac{i \mu J}{2} c_{r, j}^{\dagger}\left(c_{r+1, j}-c_{r-1, j}\right)+\text { h.c. }
\end{aligned}
$$

In order to compute the average values of the operators in Eqs.(A6), we assume that each lead $j$ is connected to a thermal reservoir at voltage bias $V_{j}$ and at temperature $T_{j}=T+\delta T_{j}$. Collecting the contributions all together, we obtain the expectation value of the electric current

$$
\begin{gathered}
I_{\mathrm{el}, j}=\left\langle j_{\mathrm{el}, j}(x, t)\right\rangle=e \sum_{\epsilon} \sum_{j^{\prime}=1}^{N}\left\{v_{p}\left[T_{j, j^{\prime}}(\epsilon)-\delta_{j, j^{\prime}}\right] f_{j^{\prime}}^{(p)}(\epsilon)+v_{p} A_{j, j^{\prime}}(\epsilon)\left[1-f_{j^{\prime}}^{(p)}(\epsilon)\right]\right. \\
\left.+v_{h} \tilde{A}_{j, j^{\prime}}(\epsilon) f_{j^{\prime}}^{(h)}(\epsilon)+v_{h}\left[\tilde{T}_{j, j^{\prime}}(\epsilon)-\delta_{j, j^{\prime}}\right]\left[1-f_{j^{\prime}}^{(h)}(\epsilon)\right]\right\}
\end{gathered}
$$

with $v_{p / h}=v_{p / h}(\epsilon)=2 J \sin \left(k_{p / h}(\epsilon)\right)$. Similarly, the thermal current is

$$
\begin{aligned}
I_{\mathrm{th}, j}= & \left\langle j_{\mathrm{th}, j}(x, t)\right\rangle=\sum_{\epsilon}(\epsilon-\mu) \sum_{j^{\prime}=1}^{N}\left\{v_{p}\left[T_{j, j^{\prime}}(\epsilon)-\delta_{j, j^{\prime}}\right] f_{j^{\prime}}^{(p)}(\epsilon)-v_{p} A_{j, j^{\prime}}(\epsilon)\left[1-f_{j^{\prime}}^{(p)}(\epsilon)\right]\right. \\
& \left.+v_{h} \tilde{A}_{j, j^{\prime}}(\epsilon) f_{j^{\prime}}^{(h)}(\epsilon)-v_{h}\left[\tilde{T}_{j, j^{\prime}}(\epsilon)-\delta_{j, j^{\prime}}\right]\left[1-f_{j^{\prime}}^{(h)}(\epsilon)\right]\right\},
\end{aligned}
$$

with the scattering coefficients given in Section II and the Fermi distribution functions for particles and holes respectively given by Eq.(5) in the main text. In the large- $\ell$ limit, we trade the sum over the energies for integrals and introduce the density of states around the
Fermi energy $\rho_{0}$. Linearizing the Fermi distribution in 
the voltage and temperature biases

$$
\begin{aligned}
& f_{j}^{(p)}(\epsilon) \approx f(\epsilon)+\left[\left(\frac{\epsilon-\mu}{k_{B} T^{2}}\right) \delta T_{j}-\frac{e V_{j}}{k_{B} T}\right] \partial_{\epsilon} f(\epsilon-\mu) \\
& f_{j}^{(h)}(\epsilon) \approx f(\epsilon)+\left[\left(\frac{\epsilon-\mu}{k_{B} T^{2}}\right) \delta T_{j}+\frac{e V_{j}}{k_{B} T}\right] \partial_{\epsilon} f(\epsilon-\mu)
\end{aligned}
$$

and employing the unitarity of the extended S-matrix, as well as the Sommerfeld expansion for the resulting integrals at temperatures $k_{B} T \ll \mu$, we obtain

$$
\begin{aligned}
I_{\mathrm{el}, j} & =-\frac{e^{2}}{2 \pi} \sum_{j^{\prime}=1}^{N}\left[\delta_{j, j^{\prime}}+C_{j, j^{\prime}}-T_{j, j^{\prime}}\right] V_{j^{\prime}} \\
I_{\mathrm{th}, j} & =\frac{\pi k_{B}^{2} T}{6} \sum_{j^{\prime}=1}^{N}\left[C_{j, j^{\prime}}+T_{j, j^{\prime}}-\delta_{j, j^{\prime}}\right] \delta T_{j^{\prime}}
\end{aligned}
$$

with the dependence on $\epsilon$ in the scattering coefficients dropped to mean that all of them are computed at $\epsilon=\mu$. From Eqs.(A10), cast in the form

$$
I_{\mathrm{el}, j}=\sum_{j^{\prime}=1}^{N} G_{j, j^{\prime}} V_{j^{\prime}}, I_{\mathrm{th}, j}=\sum_{j^{\prime}=1}^{N} K_{j, j^{\prime}} \delta T_{j^{\prime}},
$$

we obtain Eq.(7) in the main text. As a side remark, we point out that throughout the paper we can safely compute the heat current by averaging the energy current operator, rather than the heat itself. Since the two operators differ by $\sum_{j=1}^{N} V_{j} I_{\mathrm{el}, j}$, we see that it is of second order in the biases $V_{j}, \delta T_{j}$. Thus, we can safely neglect it within linear response theory.

\section{Appendix B: Electric and thermal conductance for a ballistic interacting single quantum wire}

In this Appendix we review the calculation of the electric and of the thermal conductance for a single interacting quantum wire connected to two reservoirs kept at different voltages and temperatures. Besides reviewing well-known results [13, 14, 37, and 38], we set up our formal approach to computing the CCT and the HCT. In Fig. 8 we sketch the wire connected to a left-hand reservoir, which injects into the system right-handed modes at voltage bias $V_{R}$ and at temperature $T_{R}$, and to a righthand reservoir that injects left-handed modes at voltage bias $V_{L}$ and at temperature $T_{L}$. To describe the wire, we employ a minimal model for the corresponding Hamiltonian in fermionic coordinates, $H_{\mathrm{Fer}}$, which is given by

$$
\begin{aligned}
H_{\mathrm{Fer}} & =-i v \int_{0}^{\ell} d x\left\{\psi_{R}^{\dagger}(x) \partial_{x} \psi_{R}(x)-\psi_{L}^{\dagger}(x) \partial_{x} \psi_{L}(x)\right\} \\
& +2 V \int_{0}^{\ell} d x \psi_{R}^{\dagger}(x) \psi_{R}(x) \psi_{L}^{\dagger}(x) \psi_{L}(x),
\end{aligned}
$$

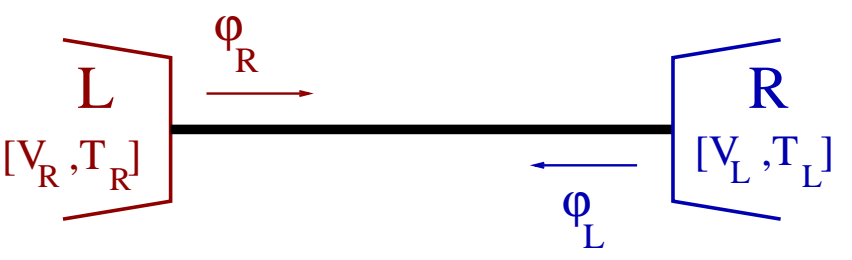

Figure 8. Sketch of a single, interacting quantum wire connected to a left-hand reservoir, which injects into the system right-handed modes at voltage bias $V_{R}$ and at temperature $T_{R}$ (colored in red), and to a right-hand reservoir that injects left-handed modes at voltage bias $V_{L}$ and at temperature $T_{L}$ (colored in blue).

with $V$ being the bulk interaction strength. Along the bosonization approach, we introduce two chiral bosonic fields $\varphi_{R}(x, t)=\varphi_{R}\left(t_{-}\right)$and $\varphi_{L}(x, t)=\varphi_{L}\left(t_{+}\right)$. Their dynamics is governed by the Hamiltonian

$$
\begin{aligned}
H_{\mathrm{Bos}, 0} & =u \int_{0}^{\ell} d x\left\{\left(\partial_{x} \varphi_{R}(x)\right)^{2}+\left(\partial_{x} \varphi_{L}(x)\right)^{2}\right\} \\
& \equiv H_{\mathrm{Bos}, 0, R}+H_{\mathrm{Bos}, 0, L}
\end{aligned}
$$

with the plasmon velocity $u=v \sqrt{1-\frac{V^{2}}{v^{2} \pi^{2}}}$. The fermion operators are represented via

$$
\begin{aligned}
& \psi_{R}(x)=\Gamma e^{\sqrt{\pi}\left[\left(\frac{1}{\sqrt{g}}+\sqrt{g}\right) \varphi_{R}(x)+\left(\frac{1}{\sqrt{g}}-\sqrt{g}\right) \varphi_{L}(x)\right]} \\
& \psi_{L}(x)=\Gamma e^{\sqrt{\pi}\left[\left(\frac{1}{\sqrt{g}}-\sqrt{g}\right) \varphi_{R}(x)+\left(\frac{1}{\sqrt{g}}+\sqrt{g}\right) \varphi_{L}(x)\right]}
\end{aligned}
$$

in which the Klein factors $\Gamma$ can be safely discarded, when bosonizing a single QW. Finally, we rewrite the densities and the current operators in terms of the chiral fields as

$$
\begin{aligned}
\rho_{\mathrm{el}}(x, t) & =e \sqrt{\frac{g}{\pi}}\left\{\partial_{x} \varphi_{R}(x, t)-\partial_{x} \varphi_{L}(x, t)\right\} \\
\rho_{\mathrm{th}}(x, t) & =u\left\{\left(\partial_{x} \varphi_{R}(x, t)\right)^{2}+\left(\partial_{x} \varphi_{L}(x, t)\right)^{2}\right\},
\end{aligned}
$$

and

$$
\begin{aligned}
& j_{\mathrm{el}}(x, t)=e u \sqrt{\frac{g}{\pi}}\left\{\partial_{x} \varphi_{R}(x, t)+\partial_{x} \varphi_{L}(x, t)\right\} \\
& j_{\mathrm{th}}(x, t)=u^{2}\left\{\left(\partial_{x} \varphi_{R}(x, t)\right)^{2}-\left(\partial_{x} \varphi_{L}(x, t)\right)^{2}\right\}
\end{aligned}
$$

In order to take into account that modes with opposite chiralities are "shot in" from the reservoirs at different voltage bias (which couple with the corresponding charge density operators), we modify the wire Hamiltonian by adding a voltage dependent "source" term. As a result, we obtain

$$
\begin{aligned}
H_{\mathrm{Bos}}\left[V_{L}, V_{R}\right] & =u \int_{0}^{\ell} d x\left\{\left(\partial_{x} \varphi_{R}(x)\right)^{2}+\left(\partial_{x} \varphi_{L}(x)\right)^{2}\right\} \\
& -e \sqrt{\frac{g}{\pi}} \int_{0}^{\ell} d x\left\{V_{R} \partial_{x} \varphi_{R}(x)-V_{L} \partial_{x} \varphi_{L}(x)\right\} \\
& \equiv H_{\mathrm{Bos}, L}\left[V_{L}\right]+H_{\mathrm{Bos}, R}\left[V_{R}\right] .
\end{aligned}
$$


Chiral modes are shot in from the reservoirs at different temperatures, as well. In order to account for the different temperature of the opposite chirality modes, given a generic operator $\mathcal{O}$ that is a functional of $\varphi_{R}(x), \varphi_{L}(x)$, we compute its thermal average $\langle\mathcal{O}\rangle$ according to

$$
\langle\mathcal{O}\rangle=\frac{\operatorname{Tr}\left[\mathcal{O} e^{-\beta_{L} H_{\mathrm{Bos}, L}\left[V_{L}\right]-\beta_{R} H_{\mathrm{Bos}, R}\left[V_{R}\right]}\right]}{\mathcal{Z}_{L}\left[V_{L}, \beta_{L}\right] \mathcal{Z}_{R}\left[V_{R}, \beta_{R}\right]},
$$

with $\beta_{R, L}=\left(k_{B} T_{R, L}\right)^{-1}$ and the partition functions for the chiral modes being given by

$$
\mathcal{Z}_{L, R}\left[V_{L, R}, \beta_{L, R}\right]=\operatorname{Tr}\left[e^{-\beta_{L, R} H_{\mathrm{Bos}, L, R}}\right] .
$$

It is customary to define the shifted chiral fields $\bar{\varphi}_{R}(x), \bar{\varphi}_{L}(x)$, according to

$$
\begin{aligned}
& \partial_{x} \bar{\varphi}_{R}(x)=\partial_{x} \varphi_{R}(x)-\frac{e}{2 u} \sqrt{\frac{g}{\pi}} V_{R} \\
& \partial_{x} \bar{\varphi}_{L}(x)=\partial_{x} \varphi_{L}(x)+\frac{e}{2 u} \sqrt{\frac{g}{\pi}} V_{L} .
\end{aligned}
$$

Taking advantage of the system homogeneity, we compute the average value of the charge- and of the thermalcurrent, $I_{e}$ and $I_{h}$, as

$$
I_{\mathrm{el} / \mathrm{th}}=\frac{1}{\ell} \int_{0}^{\ell} d x\left\langle j_{\mathrm{el} / \mathrm{th}}(x)\right\rangle .
$$

As a result, retaining only linear contributions in the voltage/temperature bias and using the relation

$$
u^{2} \int_{0}^{\ell} d x\left\langle\left(\partial_{x} \bar{\varphi}_{L / R}(x)\right)^{2}\right\rangle=\frac{1}{u} \partial_{\beta_{L / R}} \ln \mathcal{Z}_{L, R},
$$

we obtain

$$
I_{\mathrm{el}}=\frac{e^{2} g}{2 \pi}\left(V_{R}-V_{L}\right) \quad, \quad I_{\mathrm{th}}=\frac{\pi}{12 \beta_{R}^{2}}-\frac{\pi}{12 \beta_{L}^{2}} .
$$

Setting $\Delta V=V_{R}-V_{L}$ and $T_{R}=T+\frac{\Delta T}{2}, T_{R}=T-\frac{\Delta T}{2}$, the charge and the thermal conductance follow directly

$$
G=\frac{I_{\mathrm{el}}}{\Delta V}=\frac{g e^{2}}{2 \pi} \quad, \quad K=\frac{I_{\mathrm{th}}}{\Delta T}=\frac{\pi k_{B}^{2} T}{6},
$$

as derived in [37]. In this paper, it was observed that Eq.(B13) implies the violation of the WFL, as evidenced by the renormalization of the Lorenz ratio to $\mathcal{L}=L_{0} / g$. This is directly related to the peculiar nature of the electronic interaction in one-dimensional systems, that typically cannot be accounted for within the Fermi liquid picture. Nevertheless, we also note that, since the renormalization of $L_{0}$ is associated to the interaction-dependent renormalization of $G$ in a Luttinger liquid, we expect that it is washed out as soon as the 1d interacting system is connected to external Fermi liquid reservoirs, in perfect analogy with what happens to the electric conductance of a Luttinger liquid connected to noninteracting reservoirs [2, 43-45]. In Appendix $\mathrm{C}$ we show that this is the case. As we discuss in the main text, only at a junction where multi-particle scattering processes take place even in the noninteracting limit, one recovers a "genuine" violation of the WFL, that survives the presence of Fermi liquid reservoirs attached to the interacting QWs.

\section{Appendix C: Renormalization of the conductance tensors at a junction of Luttinger liquids connected to Fermi liquid reservoirs}

In this Appendix we derive the renormalization of the conductance tensor of a junction of interacting QWs connected to external Fermi liquid reservoirs. For the charge conductance, this is a well-known effect, due to the fact that, over long enough length scales and/or at low enough energies, the collective modes of the Luttinger liquid are determined by the parameters of the external leads $[2,43-45]$. We extend the analysis to the thermal conductance tensor, so to determine the effects of the Fermi liquid reservoirs directly on the Lorenz ratio.

Following [2, 43-45], we model the reservoirs in terms of a discontinuity of the Luttinger parameter in each wire at $x=\ell$. Specifically, we set

$$
\begin{aligned}
& g(x)= \begin{cases}g, & 0<x<\ell \\
g_{\text {Res }}, & \ell \leq x\end{cases} \\
& u(x)= \begin{cases}u, & 0<x<\ell \\
u_{\text {Res }}, & \ell \leq x\end{cases}
\end{aligned}
$$

In the following, we denote with $\varphi_{R / L, \text { Res, } j}$ and with $\varphi_{R / L, j}$ the chiral bosonic fields within the Fermi liquid reservoirs and within the interacting QWs, respectively. At the interface at $x=\ell$, the continuity of the fields $\phi_{j}(x)$, as well as of the charge current density operators, implies the linear relations [6]

$$
\left[\begin{array}{l}
\varphi_{R, \operatorname{Res}, j}(\ell) \\
\varphi_{L, \operatorname{Res}, j}(\ell)
\end{array}\right]=\mathbf{m} \cdot\left[\begin{array}{l}
\varphi_{R, j}(\ell) \\
\varphi_{L, j}(\ell)
\end{array}\right]
$$

between the chiral fields of the system and of the reservoirs at the contact point. The orthogonal interface transfer matrix $\mathbf{m}$ is given by

$$
\mathbf{m}=\frac{1}{2 \sqrt{g g_{\text {Res }}}}\left[\begin{array}{ll}
g_{\text {Res }}+g & g_{\text {Res }}-g \\
g_{\text {Res }}-g & g_{\text {Res }}+g
\end{array}\right] \equiv\left[\begin{array}{ll}
m_{R R} & m_{R L} \\
m_{L R} & m_{R R}
\end{array}\right] .
$$

The reservoirs inject chiral $L$ modes, whose dynamics is governed by the Luttinger Hamiltonian with parameter $g_{\text {Res }}$. Having in mind a setup in which the current measurement is performed via the reservoir $j$ (as in [57]), we need to express the outgoing modes in that reservoir in terms of the incoming ones (in any reservoir). This task is performed by a pertinent splitting matrix $\hat{\rho}$, defined via

$$
\varphi_{R, \operatorname{Res}, j}\left(t-\frac{x}{u_{\operatorname{Res}}}\right)=\sum_{j^{\prime}=1}^{3} \hat{\rho}_{j, j^{\prime}} \varphi_{L, \operatorname{Res}, j^{\prime}}\left(t+\frac{x}{u_{\text {Res }}}\right)
$$

Combining the interface transfer matrix Eq.(C3) with the junction splitting matrix Eq.(16), we obtain

$$
\hat{\rho}_{j, j^{\prime}}=\sum_{j^{\prime \prime}=1}^{N}\left[M_{R}\right]_{j, j^{\prime \prime}}\left[M_{L}^{-1}\right]_{j^{\prime \prime}, j^{\prime}},
$$


with

$$
\begin{aligned}
& {\left[M_{R}\right]_{j, j^{\prime}}=m_{R R} \rho_{j, j^{\prime}}+m_{R L} \delta_{j, j^{\prime}}} \\
& {\left[M_{L}\right]_{j, j^{\prime}}=m_{L R} \rho_{j, j^{\prime}}+m_{L L} \delta_{j, j^{\prime}} .}
\end{aligned}
$$

We therefore conclude that the effect of the reservoirs is taken into account by substituting the splitting matrix $\rho$ with $\hat{\rho}$ defined in (C5). Substituting the parametrization Eqs. (26) and (27) for the charge-conserving junction, one checks that, for the $N=3, \mathbb{Z}_{3}$ invariant junction, the resulting splitting matrix is obtained by simply substituting $g$ with $g_{\text {Res }}$ in the splitting matrix of the junction disconnected from the reservoirs, that is

$$
\hat{\rho}(\vartheta)=\left.\rho(\vartheta)\right|_{g \rightarrow g_{\text {Res }}}
$$

At variance, when charge conservation holds but $\mathbb{Z}_{3}$ symmetry is broken, Eq.(31), the splitting matrices $\rho_{B}$ are independent of $g$ and of $g_{\text {Res }}$, so one trivially obtains the same result with, or without, connecting the junction to Fermi liquid reservoirs.

\section{Appendix D: Green-Keldysh functions of bosonic operators}

In this appendix, we review the Keldysh-Green functions involving chiral bosonic vertex operators, in the time, as well as in the frequency domain.

We start with the Keldysh path-ordered correlation function of the chiral fields

$$
\begin{aligned}
& g_{\eta_{1} \eta_{2} ; j}^{-\nu}\left(t_{1}-t_{2}+\frac{x_{1}-x_{2}}{u}\right)= \\
& \left\langle\mathcal{T}_{K} e^{i \sqrt{4 \pi \nu} \varphi_{j, \eta_{1}}\left(t_{1}+x_{1} / u ; \eta_{1}\right)} e^{-i \sqrt{4 \pi \nu} \varphi_{j, \eta_{2}}\left(t_{2}+x_{2} / u ; \eta_{2}\right)}\right\rangle
\end{aligned}
$$

with the Keldysh indices $\eta_{1,2}= \pm 1$ and $\mathcal{T}_{K}$ denoting the ordering operator along the Keldysh path. The Green's functions are [98]

$$
\begin{aligned}
& g_{++, j}^{-\nu}\left(t+\frac{x}{u}\right)=\left\{\frac{\beta_{j}}{\pi} \sin \left[\frac{\pi}{\beta_{j}}\left(i\left(t+\frac{x}{u}\right) \operatorname{sgn}(t)+\tau_{c}\right)\right]\right\}^{-\nu} \\
& g_{-+, j}^{-\nu}\left(t+\frac{x}{u}\right)=\left\{\frac{\beta_{j}}{\pi} \sin \left[\frac{\pi}{\beta_{j}}\left(i\left(t+\frac{x}{u}\right)+\tau_{c}\right)\right]\right\}^{-\nu}(\mathrm{D} 2)
\end{aligned}
$$

with $\quad g_{-\eta_{1}-\eta_{2}, j}^{-\nu}\left(t+\frac{x}{u}\right)=\left[g_{\eta_{1} \eta_{2}, j}^{-\nu}\left(-t-\frac{x}{u}\right)\right] \quad$ and $\tau_{c} \sim D_{0}^{-1}$. Their Fourier transforms

$$
\tilde{g}_{\eta_{1} \eta_{2}, j}^{-\nu}(\omega)=\int d t e^{i \omega t} g_{\eta_{1} \eta_{2}, j}^{-\nu}(t)
$$

are derived in detail in [98]. Here we quote the result

$$
\tilde{g}_{\eta_{1} \eta_{2}, j}^{-\nu}(\omega)=c_{\eta_{1} \eta_{2}, j}^{-\nu}(\omega) d_{j}^{-\nu}(\omega)
$$

with, denoting by $\Gamma(z)$ the Euler's $\Gamma$ function,

$$
\begin{gathered}
d_{j}^{-\nu}(\omega)=\frac{\beta_{j}^{1-\nu}}{(2 \pi)^{1-\nu} \Gamma(\nu)}\left|\Gamma\left(\frac{\nu}{2}+i \frac{\beta_{j} \omega}{2 \pi}\right)\right|^{2}, \\
c_{ \pm \pm, j}^{-\nu}(\omega)=\frac{2 \cosh \frac{\beta_{j} \omega}{2}}{1+e^{ \pm i \pi \nu}} \quad, \quad c_{\mp \pm, j}^{-\nu}(\omega)=e^{ \pm \frac{\beta_{j} \omega}{2}} .
\end{gathered}
$$

Eq. (D1) is easily generalized to a multipoint correlation function. In particular, we need the multiple contraction

$$
g_{\left\{\eta_{n}\right\}_{n} ; j}^{\left\{\alpha_{n}\right\}_{n} ; N ;-\nu}\left(\left\{t_{n}\right\}_{n}\right)=\delta_{\sum_{n} \alpha_{n}, 0} \prod_{l<m} g_{\eta_{l}, \eta_{m} ; j}^{-\alpha_{l} \alpha_{m} \nu}\left(t_{l}-t_{m}\right)
$$

It is also convenient to define the functions

$$
\xi_{ \pm, j}(u t+x)=\frac{\pi}{i \beta_{j}} \operatorname{coth}\left[\frac{\pi}{\beta_{j}}\left(t+\frac{x}{u} \mp i \tau_{c}\right)\right]
$$

whose Fourier transforms are

$$
\tilde{\xi}_{ \pm, j}(\omega)=\int d t e^{i \omega t} \xi_{ \pm, j}(t)= \pm \frac{2 \pi e^{\mp \omega \tau_{c}}}{1-e^{\mp \beta_{j} \omega}} .
$$

We compute the correlation functions of chiral fields including insertions of chiral current operators using [98]

$$
\begin{aligned}
g_{\eta,\left\{\eta_{n}\right\}_{n} ; j}^{\left\{\alpha_{n}\right\}_{n},-\nu}\left(t+\frac{x}{u} ;\left\{t_{n}\right\}_{n}\right) & =\left\langle\mathcal{T}_{K} \partial_{x} \varphi_{j, \eta}\left(t+\frac{x}{u}\right) \prod_{l=1}^{N} e^{i \alpha_{l} \sqrt{4 \pi \nu} \varphi_{j, \eta_{l}}\left(t_{l}\right)}\right\rangle= \\
& =\frac{\sqrt{\nu \pi}}{2 i u \beta_{j}} \sum_{l=1}^{N} \alpha_{l} \operatorname{coth}\left[\frac{\pi}{\beta_{j}}\left(t-t_{l}+\frac{x}{u}-i \tau_{c} \sigma_{\eta \eta_{l}}\left(t-t_{l}\right)\right)\right] g_{\left\{\eta_{n}\right\}_{n} ; j}^{\left\{\alpha_{n}\right\}_{n},-\nu}\left(t_{1}-t_{2}\right)
\end{aligned}
$$




$$
\begin{aligned}
& g_{\eta \eta^{\prime},\left\{\eta_{n}\right\}_{n} ; j}^{\{\alpha\},-\nu}\left(t+\frac{x}{u} ; t^{\prime}+\frac{x^{\prime}}{u} ;\left\{t_{n}\right\}_{n}\right)=\left\langle\mathcal{T}_{K} \partial_{x} \phi_{j, \eta}\left(t+\frac{x}{u}\right) \partial_{x^{\prime}} \phi_{j, \eta^{\prime}}\left(t^{\prime}+\frac{x^{\prime}}{u}\right) \prod_{l=1}^{N} e^{i \alpha_{l} \sqrt{4 \pi \nu} \varphi_{j, \eta_{l}}\left(t_{l}\right)}\right\rangle=(\mathrm{D} 11) \\
& =-g_{\left\{\eta_{l}\right\}_{l} ; j}^{-\nu}\left(\left\{t_{i}\right\}\right) \sum_{l, l^{\prime}=1}^{N} \frac{\alpha_{l} \alpha_{l^{\prime}} \nu \pi}{4 u^{2} \beta_{j}^{2}} \operatorname{coth}\left[\frac{\pi}{\beta_{j}}\left(t-t_{l}+\frac{x}{u}-i \tau_{c} \sigma_{\eta \eta_{l}}\left(t-t_{l}\right)\right)\right] \operatorname{coth}\left[\frac{\pi}{\beta_{j}}\left(t^{\prime}-t_{l^{\prime}}+\frac{x^{\prime}}{u}-i \tau_{c} \sigma_{\eta^{\prime} \eta_{l^{\prime}}}\left(t-t_{l^{\prime}}\right)\right)\right]
\end{aligned}
$$

where

$$
\sigma_{\eta_{1} \eta_{2}}\left(t_{1}-t_{2}\right)=\eta_{2} \theta\left(t_{1}-t_{2}\right)-\eta_{1} \theta\left(t_{2}-t_{1}\right) .
$$

All the fields in the above contractions are in equilibrium with the $j$-th reservoir at temperature $\beta_{j}^{-1}$.

\section{Appendix E: General expression for the leading corrections to the fixed point conductance}

In this Appendix we provide a general formula for the leading corrections to the FP conductance tensors of the junction, given the corresponding leading boundary operator and the splitting matrix Eq.(16). Consistently with our analysis in the main text, in full generality, we assume that the leading boundary operator is realized as a combination of vertex operators depending on pertinent linear combinations of the fields $\varphi_{j}(0)$, defined in Eq.(17). Also, we assume full symmetry between the various channels, which allows us to assume an over-all constant independent of the specific vertex operator. Thus, we set

$$
H_{B}=-2 h \sum_{k<l=1}^{N} \cos \left[\sqrt{4 \pi} \sum_{j=1}^{N} \alpha_{j}^{k, l} \varphi_{j}(0)\right],
$$

with $\alpha_{j}^{k, l}$ being a coefficient that explicitly depends on $g$. For instance, at the DFP, consistently with the boundary interactions of the junction (47) and of the TKM (48) for $N=3$, we set $\alpha_{j}^{k, l}=g^{-\frac{1}{2}}\left\{\delta_{k, j}-\delta_{l, j+1}\right\}$.

In our picture, the external reservoirs inject into the leads left-handed modes biased at a voltage $V_{j}$. We account for this by the shift in Eq.(22), which provides an explicit dependence on time in the leading boundary operator

$$
H_{B}=-2 h \sum_{k<l}^{N} \cos \left[\sqrt{4 \pi} \sum_{j=1}^{N} \alpha_{j}^{k, l}\left(\bar{\varphi}_{j}(0)+e \sqrt{\frac{g}{4 \pi}} V_{j} t\right)\right]
$$

The electric current operator takes the form

$$
\begin{aligned}
j_{\mathrm{el}, j}(x, t)=e u & \sqrt{\frac{g}{\pi}}\left[\partial_{x} \bar{\varphi}_{j}\left(t_{+}\right)-\sum_{j^{\prime}=1}^{N} \rho_{j^{\prime}, j} \partial_{x} \bar{\varphi}_{j}\left(t_{-}\right)\right] \\
& +\frac{e^{2} g}{2 \pi} \sum_{j^{\prime}=1}^{N}\left\{\rho_{j, j^{\prime}}-\delta_{j, j^{\prime}}\right\} V_{j^{\prime}} .
\end{aligned}
$$

We have already shown that, within linear response theory, the FP electric conductance is given by Eq.(23), which is perfectly consistent with the result in Eq.(E3). Due to the neutrality constraint on the vertex operators, no corrections arise to first order in the perturbation Eq.(E2). Using the Keldysh formalism up to second order, we obtain the expectation value of the electric and thermal currents in the form

$$
I_{\mathrm{el} / \mathrm{th}, j}=h^{2} \sum_{\eta_{1}, \eta_{2}} \eta_{1} \eta_{2} \int d t_{1} d t_{2} \sum_{k<l} \prod_{j_{1}=1}^{N}\left\langle\mathcal{T}_{K} j_{\mathrm{el} / \mathrm{th}, j}(x, t) e^{-i \sqrt{4 \pi} \alpha_{j_{1}}^{k, l} \varphi_{j_{1}, \eta_{1}}\left(t_{1}\right)} e^{i \sqrt{4 \pi} \alpha_{j_{1}}^{k, l} \varphi_{j_{1}, \eta_{2}}\left(t_{2}\right)}\right\rangle e^{i e \sqrt{g} \alpha_{j_{1}}^{k, l} V_{j_{1}}\left(t_{1}-t_{2}\right)}+\mathrm{h} . \mathrm{c} .
$$

In Eq.(E4) $\eta_{1,2}= \pm 1$ label the branch of the Keldysh contour and the current operator lies, by convention, on the upper branch $(\eta=+)$.

We assume an homogeneous temperature throughout the system and turn on a potential bias on lead $j_{b} \neq j$. As we work close to the charge neutrality point, the Seebeck and Peltier coefficients vanish and only an electric current is generated. Using Eqs. (E3) and (D10) and their Fourier transforms Eqs.(D9) and (D4), we obtain

$$
\begin{aligned}
I_{\mathrm{el}, j}= & \frac{e h^{2} \sqrt{g}}{4 \pi} \sum_{k<l=1}^{N} \sum_{\eta_{1}, \eta_{2}= \pm 1} \eta_{1} \eta_{2} \sum_{j^{\prime}=1}^{N} \rho_{j, j^{\prime}} \alpha_{j^{\prime}}^{k, l} \times \\
& \sum_{s= \pm 1} s\left[\tilde{\xi}_{\eta_{1}, j^{\prime}}(0)-\tilde{\xi}_{\eta_{2}, j^{\prime}}(0)\right] \tilde{g}_{\eta_{1} \eta_{2} ; j}^{-\nu}\left(s_{2} e \sqrt{g} \alpha_{j_{b}}^{k, l} V_{j_{b}}\right) .
\end{aligned}
$$

Here $\nu=\sum_{m=1}^{N}\left(\alpha_{m}^{k, l}\right)^{2}$. Finally, retaining only contributions that are linear in the applied biases, we obtain the 
simple expression

$I_{\mathrm{el}, j}=2 \pi g e^{2} \tilde{h}^{2}\left(2 \pi k_{B} T\right) \frac{\Gamma^{2}(\nu / 2)}{\Gamma(\nu)} \sum_{k<l=1}^{N} \sum_{j^{\prime}=1}^{N} \rho_{j, j^{\prime}} \alpha_{j^{\prime}}^{k, l} \alpha_{j_{b}}^{k, l}$,

with the dimensionless coupling $\tilde{h}(D)=\frac{h}{D_{0}}\left(\frac{D}{D_{0}}\right)^{-1+\frac{\nu}{2}}$.

We now examine the thermal current at charge neutrality Eq. (21). We start again from Eq. (E4) and set $V_{j}=0$ in all leads, but allow for a different temperature $T_{j}$ on each lead $j=1, \ldots, N$. We then make use of Eqs. (D2) and (D8), as well as of the Fourier transforms Eq. (D9), to write the expectation value as $I_{\mathrm{th}, j}=I_{\mathrm{th}, j}^{(A)}+I_{\mathrm{th}, j}^{(B)}$, in which

$$
\begin{aligned}
& I_{\mathrm{th}, j}^{(A)}=\frac{h^{2}}{4 \pi} \sum_{k<l} \sum_{\eta_{1}, \eta_{2}= \pm 1} \eta_{1} \eta_{2} \sum_{j_{a}, j_{b}} \rho_{j, j_{a}} \rho_{j, j_{b}} \alpha_{j_{a}}^{k, l} \alpha_{j_{b}}^{k, l} \quad(\mathrm{E} 6) \\
& \quad \times \tilde{\Gamma}_{\eta_{1}, \eta_{2}}^{\left\{\alpha^{k, l}\right\}}(0) \int \frac{d \omega}{2 \pi}\left[\tilde{\xi}_{\eta_{1}, j_{a}}(\omega) \tilde{\xi}_{\eta_{1}, j_{b}}(-\omega)+\left(\eta_{1} \rightarrow \eta_{2}\right)\right], \\
& I_{\mathrm{th}, j}^{(B)}=\frac{h^{2}}{4 \pi} \sum_{k<l} \sum_{\eta_{1}, \eta_{2}= \pm 1} \eta_{1} \eta_{2} \sum_{j_{a}, j_{b}} \rho_{j, j_{a}} \rho_{j, j_{b}} \alpha_{j_{a}}^{k, l} \alpha_{j_{b}}^{k, l} \quad(\mathrm{E} 7) \\
& \quad \times \int \frac{d \omega}{2 \pi} \tilde{\Gamma}_{\eta_{1}, \eta_{2}}^{\left\{\alpha^{k, l}\right\}}(\omega)\left[\tilde{\xi}_{\eta_{1}, j_{a}}(\omega) \tilde{\xi}_{\eta_{2}, j_{b}}(-\omega)+\left(j_{a} \leftrightarrow j_{b}\right)\right] .
\end{aligned}
$$

The function $\tilde{\Gamma}$ originates the contractions of the derivative of the fields $j_{a}$ and $j_{b}$ with the corresponding perturbation at second order. It is labeled by the coefficients $\left\{\alpha_{1}^{k, l}, \ldots, \alpha_{N}^{k, l}\right\}$ and stands for

$$
\tilde{\Gamma}_{\eta_{1}, \eta_{2}}^{\left\{\alpha^{k, l}\right\}}(t)=\prod_{m=1}^{N} g_{\eta_{1} \eta_{2} ; m}^{-\left(\alpha_{m}^{k, l}\right)^{2}}(t)
$$

By going along the derivation of Appendix D, it is easy to verify the following identities

$$
\begin{aligned}
& \sum_{\sigma= \pm} \tilde{\xi}_{ \pm, j_{a}}(\sigma \omega) \tilde{\xi}_{ \pm, j_{b}}(-\sigma \omega)=-\frac{2 \pi^{2} \cosh \frac{\left(\beta_{a}-\beta_{b}\right) \omega}{2}}{\sinh \frac{\beta_{a} \omega}{2} \sinh \frac{\beta_{b} \omega}{2}}, \quad(\mathrm{E} 9) \\
& \sum_{\eta= \pm} \tilde{\xi}_{\eta, j_{a}}( \pm \eta \omega) \tilde{\xi}_{-\eta, j_{b}}(\mp \eta \omega) \\
& =\sum_{\sigma= \pm} \tilde{\xi}_{+, j_{a}}(\sigma \omega) \tilde{\xi}_{+, j_{b}}(-\sigma \omega) \mp 2 \pi\left[\tilde{\xi}_{ \pm, j_{a}}(\omega)+\tilde{\xi}_{ \pm, j_{b}}(\omega)\right]
\end{aligned}
$$

with $\beta_{a}=\left(k_{B} T_{a}\right)^{-1}$. Using Eq. (E9), together with the relation

$$
\tilde{\Gamma}_{\eta_{1}, \eta_{2}}^{\left\{\alpha^{k, l}\right\}}(\omega=0)=\tilde{\Gamma}_{-\eta_{1},-\eta_{2}}^{\left\{\alpha^{k, l}\right\}}(\omega=0),
$$

one concludes that Eq. (E6) is identically zero. In addition to that, we can exploit Eq. (E10) to rewrite Eq.(E7) in the simpler form

$$
I_{\mathrm{th}, j}=I_{\mathrm{th}, j}^{(B)}=h^{2} \sum_{k<l} \sum_{j_{a}, j_{b}} \rho_{j, j_{a}} \rho_{j, j_{b}} \alpha_{j_{a}}^{k, l} \alpha_{j_{b}}^{k, l} \mathcal{A}_{j_{a}}^{\left\{\alpha^{k, l}\right\}},
$$

with

$$
\mathcal{A}_{j}^{\left\{\alpha^{k, l}\right\}}=\int \frac{d \omega}{2 \pi}\left[\tilde{\Gamma}_{-,+}^{\left\{\alpha^{k, l}\right\}}(\omega) \tilde{\xi}_{-, j}(\omega)-\tilde{\Gamma}_{+,-}^{\left\{\alpha^{k, l}\right\}}(\omega) \tilde{\xi}_{+, j}(\omega)\right] .
$$

Using the explicit Fourier transforms Eqs. (D4) and (D6), together with (D9) and the symmetry of the system under exchange of wires, it is possible to more explicitly rewrite the integral Eq.(E13) as

$$
\begin{aligned}
& \mathcal{A}_{j}^{\left\{\alpha^{k, l}\right\}}=-\int d \omega_{1} \ldots d \omega_{N} \frac{\sinh \frac{\sum_{s \neq j}\left(\beta_{j}-\beta_{s}\right) \omega_{l}}{2}}{\sinh \frac{\beta_{j} \omega_{j}}{2}} \\
& \times d_{j}^{-\left(\alpha_{j}^{k l}\right)^{2}}\left(\omega_{j}-\omega_{1} \ldots \hat{\omega}_{j} \ldots-\omega_{N}\right) \prod_{m \neq j} d_{m}^{-\left(\alpha_{m}^{k l}\right)^{2}}\left(\omega_{m}\right) .
\end{aligned}
$$

Here the ^ symbol denotes omission. It is now easy to verify that the thermal current vanishes in the absence of temperature gradients, i.e., whenever $\beta_{1}=\ldots=\beta_{N}=\beta$.

Focusing on wire $j_{0}$ (which implies no loss of generality due to the symmetry of the system) and assuming that all the remaining $N-1$ subsystems are in equilibrium at the same temperature $1 / \beta$, it is then convenient to define the function

$$
\tilde{\Gamma}_{\eta_{1}, \eta_{2}}^{\left\{\alpha^{k, l} ; \hat{j}_{0}\right\}}(t)=\prod_{m \neq j_{0}} \tilde{g}_{\eta_{1} \eta_{2} ; m}^{-\left(\alpha_{m}^{k, l}\right)^{2}}(t)
$$

where we have denoted by $\left\{\alpha ; \hat{j}_{0}\right\}$ the set $\left\{\alpha^{k, l}\right\}$, from which the element $\alpha_{j_{0}}^{k, l}$ has been removed. One can invert the Fourier transform (D4) and see directly from the definition (D2) that

$$
\tilde{\Gamma}_{\mp \pm}^{\left\{\alpha^{k, l} ; \hat{j}_{0}\right\}}(\omega)=e^{ \pm \frac{\beta \omega}{2}} d_{j}^{-\sum_{m \neq j_{0}}\left(\alpha_{m}^{k, l}\right)^{2}}(\omega)
$$

With the aid of this expression, in the presence of a small temperature bias $\beta \rightarrow \beta_{j_{0}}=\beta+\delta \beta_{j_{0}}$ on lead $j_{0}$ only, the integral $\mathcal{A}_{j}^{\left\{\alpha^{k, l}\right\}}$ in Eq.(E13) is conveniently rewritten as

$$
\begin{aligned}
& \mathcal{A}_{j}^{\left\{\alpha^{k, l}\right\}}=\sum_{\eta= \pm} \int \frac{d \omega d \omega_{0}}{2 \pi \eta} \tilde{g}_{\eta,-\eta}^{-\alpha_{j_{0}}}\left(\omega_{0}-\omega\right) \tilde{\Gamma}_{\eta,-\eta}^{\left\{\alpha^{k, l} ; \hat{j}_{0}\right\}}(\omega) \tilde{\xi}_{\eta, j}\left(\omega_{0}\right) \\
& \approx-\delta \beta_{j_{0}} \sum_{\eta= \pm} \int d \omega d \omega_{0} \frac{\omega d_{j_{0}}(\omega)}{\sinh \frac{\beta \omega_{0}}{2}} d_{j}^{-\sum_{m \neq j_{0}}\left(\alpha_{m}^{k, l}\right)^{2}}\left(\omega_{0}-\omega\right)
\end{aligned}
$$

Gathering all the contributions, the thermal current response in leg $j$ under a change of temperature in leg $j_{0}$ takes the form

$$
I_{\mathrm{th}, j}=\frac{\pi^{2} k_{B}}{\beta} \sum_{k<l} \sum_{j_{a}, j_{b}} \frac{\rho_{j, j_{a}} \rho_{j, j_{b}} \alpha_{j_{a}}^{k, l} \alpha_{j_{b}}^{k, l} \tilde{h}^{2}\left(\frac{2 \pi}{\beta}\right) \delta T_{j_{0}} \Phi_{j_{0}}^{\left\{\alpha^{k, l}\right\}}}{\Gamma\left[\left(\alpha_{j_{0}}^{k l}\right)^{2}\right] \Gamma\left[\sum_{r \neq j_{0}}\left(\alpha_{r}^{k l}\right)^{2}\right]}
$$

with $\delta T_{j_{0}}=T_{j_{0}}-T$, and

$$
\begin{aligned}
& \Phi_{j_{0}}^{\left\{\alpha^{k, l}\right\}}=\int d z d w \frac{w}{\sinh (\pi z)} \\
& \times\left|\Gamma\left(\frac{\left(\alpha_{j_{0}}^{k, l}\right)^{2}}{2}+i w\right) \Gamma\left(\frac{\sum_{m \neq j_{0}}\left(\alpha_{m}^{k, l}\right)^{2}}{2}+i(z-w)\right)\right|^{2}
\end{aligned}
$$


With Eqs.(E6,E17) one can compute the explicit form of the coefficients entering the leading corrections to the fixed point value of the CCT and of the HCT. In order to improve the perturbative result, the running coupling strengths may be inserted instead of the bare ones in front of the right-hand side of Eqs.(E6,E17). Within a straightforward generalization of the formalism we develop here, it is possible to address the case in which the leading boundary operator is made out of a linear combination of vertex operators with different coefficients. Using the values of $\alpha_{m}^{k, l}$ from Section V A we obtain the expressions (50), (51) and (53).

Finally, we quote the identities

$$
\begin{aligned}
& \Gamma\left(\frac{1}{2}+i z\right) \Gamma\left(\frac{1}{2}-i z\right)=\frac{\pi}{\cosh (\pi z)}, \\
& \int d z \frac{\pi}{\sinh (\pi z) \cosh (\pi(z-w))}=\frac{2 \pi w}{\cosh (\pi w)}, \\
& \Gamma\left(z^{*}\right)=\Gamma(z)^{*}, \quad \int d w \frac{w^{2}}{\cosh ^{2}(\pi w)}=\frac{1}{6 \pi}
\end{aligned}
$$

which are used to obtain an explicit expression of the Lorenz ratio in the main text.

\section{Appendix F: Boundary renormalization of the Topological Kondo model}

In this Appendix we provide more details about the renormalization of the boundary term Eq.(48). To begin with, we recall that the differences between the running couplings associated to the $J_{k, l}$ are systematically washed out along the RG trajectories [27]. This is encoded in the perturbative RG equations for the running couplings $\mathcal{J}_{k, l}(D) \equiv J_{k, l}(D)^{-1+\frac{1}{g}}$. Within $\epsilon$ expansion, with $0<$ $\epsilon\left(=1-g^{-1}\right) \ll 1$, these are given by $[32,33,87$, and 88$]$

$$
\frac{d \mathcal{J}_{k, l}}{d \ln \left(\frac{D_{0}}{D}\right)}=\epsilon \mathcal{J}_{k, l}+2 \sum_{a(\neq k, l)=1}^{N} \mathcal{J}_{k, a} \mathcal{J}_{a, l},
$$

Based on the above observation, in order to discuss the flow toward the $D^{N-1}$ fixed point, we consider the symmetric version of Eq.(F1), in which all the $\mathcal{J}_{j, k}$ are taken equal to each other and equal to $\mathcal{J}$, so to recover a single $\mathrm{RG}$ equation

$$
\frac{d \mathcal{J}}{d \ln \left(\frac{D_{0}}{D}\right)}=\epsilon \mathcal{J}+2(N-2) \mathcal{J}^{2},
$$

which is solved by

$$
\mathcal{J}(D)=\frac{\epsilon \mathcal{J}_{0}\left(\frac{D_{0}}{D}\right)^{\epsilon}}{\epsilon+2(N-2) \mathcal{J}_{0}-2(N-2) \mathcal{J}_{0}\left(\frac{D_{0}}{D}\right)^{\epsilon}},
$$

with $\mathcal{J}_{0}=\mathcal{J}\left(D=D_{0}\right)$. Identifying the running energy scale $D$ in Eq.(F3) with (the Boltzmann constant times) the temperature $T$ we see that, due to the relevance of the boundary interaction for $g \geq 1$, the system crosses over to the strongly coupled regime at a crossover temperature $T_{*}$, determined by the condition that the denominator of the right-hand side of Eq.(F3) becomes equal to 0 at $D=D_{*}=k_{B} T_{*}$. Accordingly, we obtain

$$
\epsilon^{-1}\left\{\left(\frac{D_{0}}{k_{B} T_{*}}\right)^{\epsilon}-1\right\}=\frac{1}{2(N-2) \mathcal{J}_{0}}
$$

which, for $\epsilon \rightarrow 0$, reduces to the "standard" Kondo result

$$
\ln \left(\frac{D_{0}}{k_{B} T_{*}}\right)=1+\frac{1}{2(N-2) \mathcal{J}_{0}} .
$$

At scales $T \leq T_{*}$ the system enters the strongly coupled regime.

In the presence of a nonvanishing phase $\chi$ Eq.(48) generalizes to the set of RG equations for the running parameters $\mathcal{J}(D), \chi(D)$ given by $[25]$

$$
\begin{aligned}
& \frac{d \mathcal{J}}{d \ln \left(\frac{D_{0}}{D}\right)}=\epsilon \mathcal{J}+2 \cos (\chi) \mathcal{J}^{2} \\
& \frac{d \chi}{d \ln \left(\frac{D_{0}}{D}\right)}=-2 \sin (\chi) \mathcal{J} .
\end{aligned}
$$

Eqs.(F6) typically emerge when considering the RG approach to the boundary interaction at a junction of three bosonic, one-dimensional interacting system. To construct the splitting matrix $\rho$, we first minimize the TKM boundary interaction Hamiltonian in Eq.(48), rewritten in terms of $\Phi$ and of the $\xi_{a}$ defined in Eq.(36). Then, we impose Dirichlet boundary conditions by pinning $\xi_{a}(0)$, $\forall a=1, \ldots, N-1$ (note that $\Phi$ decouples from the boundary interaction, due to the total charge conservation [2]).

As discussed in sec. VB, the system possesses two strong-coupling FPs in certain parameter ranges, when the boundary potential created by the interaction terms dominates over the kinetic part of the Hamiltonian. The corresponding conformal boundary conditions are, therefore, obtained by imposing Dirichlet boundary conditions at $x=0$ on the fields $\xi_{1}(x), \xi_{2}(x)$ in Eq.(48), which take values lying at the sites of a triangular/hexagonal lattice. The operator driving the system away from a fixed point described by Dirichlet boundary conditions on the $\xi_{a}$ corresponds to a combination of instanton operators encoding "jumps" between sites of the lattice of the minima.

The $D^{2} \mathrm{FP}$, or topological Kondo FP, extensively discussed in literature, is described by a triangular lattice. The $\hat{D}^{2} \mathrm{FP}$, instead, is described by requiring that the values of $\left(\xi_{1}(0), \xi_{2}(0)\right)$ minimizing the boundary potential span a honeycomb lattice (determined by two interpenetrating "rotated" triangular lattices [25 and 95]). Due to the reduced distance between the sites of the lattice of the minima, "short instanton" operators emerge, with a lower dimension than the boundary operators at the $D^{2} \mathrm{FP}$. The boundary conditions at the $\hat{D}^{2} \mathrm{FP}$ are the same as at the $D^{2} \mathrm{FP}$, so, we readily conclude that 
$\rho_{\hat{D}^{2}}=\rho_{D^{2}}$. The leading boundary interaction at the $\hat{D}^{2} \mathrm{FP}$ is given by [25 and 95]

$\hat{\tilde{H}}_{\mathrm{TK}, 2}=-2 \hat{h} \sum_{j=1}^{3} e^{i \frac{4 \sqrt{\pi g}}{3 \sqrt{3}}\left(2 \tilde{\varphi}_{j}(0)-\tilde{\varphi}_{j+1}(0)-\tilde{\varphi}_{j-1}(0)\right)} \tau^{+}+$h.c..

Following [25 and 95], in Eq.(F7) we have introduced an effective isospin operator $\vec{\tau}$. We have done so in order to take into account that the honeycomb lattice of min- ima at the $\hat{D}^{2} \mathrm{FP}$ is made out of the interpenetration of two inequivalent triangular lattices, say A and B. Any short instanton originating from sublattice $\mathbf{A}$ ends into one of the three nearest neighboring sites of sublattice $\mathbf{B}$, and vice versa. Thus, having arbitrarily associated the eigenvalue +1 of $\tau_{z}$ to states living over sublattice $\mathbf{A}$ and the eigenvalue -1 to states living over sublattice $\mathbf{B}$, we recover the final expression in Eq.(F7).
* domenico.giuliano@fis.unical.it

$\dagger$ buccheri@hhu.de

${ }^{1}$ C. Chamon, M. Oshikawa, and I. Affleck, Junctions of three quantum wires and the dissipative Hofstadter model, Phys. Rev. Lett. 91, 206403 (2003).

2 M. Oshikawa, C. Chamon, and I. Affleck, Junctions of three quantum wires, J. Stat. Mech. Theory Exp. 2006, P02008 (2006).

3 S. Lal, S. Rao, and D. Sen, Junction of several weakly interacting quantum wires: A renormalization group study, Phys. Rev. B 66, 165327 (2002).

4 S. Chen, B. Trauzettel, and R. Egger, Landauertype transport theory for interacting quantum wires: Application to carbon nanotube $\mathrm{Y}$ junctions, Phys. Rev. Lett. 89, 226404 (2002).

5 B. Bellazzini and M. Mintchev, Quantum fields on star graphs, J. Phys. A Math. Theor. 39, 11101 (2006).

6 C.-Y. Hou, A. Rahmani, A. E. Feiguin, and C. Chamon, Junctions of multiple quantum wires with different Luttinger parameters, Phys. Rev. B 86, 075451 (2012).

7 D. Giuliano and A. Nava, Dual fermionic variables and renormalization group approach to junctions of strongly interacting quantum wires, Phys. Rev. B 92, 125138 (2015).

8 Z. Shi and I. Affleck, Fermionic approach to junctions of multiple quantum wires attached to Tomonaga-Luttinger liquid leads, Phys. Rev. B 94, 035106 (2016).

9 S.-i. Tomonaga, Elementary theory of quantummechanical collective motion of particles, ii, Progress of Theoretical Physics 13, 482 (1955).

$10 \mathrm{~J}$. M. Luttinger, An exactly soluble model of a many-fermion system, Journal of Mathematical Physics 4, 1154 (1963).

11 F. D. M. Haldane, 'Luttinger liquid theory' of one-dimensional quantum fluids. i. properties of the Luttinger model and their extension to the general 1d interacting spinless Fermi gas, Journal of Physics C: Solid State Physics 14, 2585 (1981).

12 F. D. M. Haldane, Effective harmonic-fluid approach to low-energy properties of one-dimensional quantum fluids, Phys. Rev. Lett. 47, 1840 (1981).

13 C. L. Kane and M. P. A. Fisher, Transport in a one-channel Luttinger liquid, Phys. Rev. Lett. 68, 1220 (1992).

14 C. L. Kane and M. P. A. Fisher, Transmission through barriers and resonant tunneling in an interacting onedimensional electron gas, Phys. Rev. B 46, 15233 (1992).

15 C. Nayak, M. P. A. Fisher, A. W. W. Ludwig, and H. H. Lin, Resonant multilead point-contact tunneling, Phys. Rev. B 59, 15694 (1999).

16 S. Eggert and I. Affleck, Magnetic impurities in half-integer-spin Heisenberg antiferromagnetic chains, Phys. Rev. B 46, 10866 (1992).

17 L. I. Glazman and A. I. Larkin, New quantum phase in a one-dimensional Josephson array, Phys. Rev. Lett. 79, 3736 (1997).

18 D. Giuliano and P. Sodano, Effective boundary field theory for a Josephson junction chain with a weak link, Nucl. Phys. B 711, 480 (2005).

19 M. A. Cazalilla, R. Citro, T. Giamarchi, E. Orignac, and M. Rigol, One dimensional bosons: From condensed matter systems to ultracold gases, Rev. Mod. Phys. 83, 1405 (2011).

20 A. M. Tsvelik, Majorana fermion realization of a twochannel Kondo effect in a junction of three quantum Ising chains, Phys. Rev. Lett. 110, 147202 (2013).

21 A. M. Tsvelik and W.-G. Yin, Possible realization of a multichannel Kondo model in a system of magnetic chains, Phys. Rev. B 88, 144401 (2013).

22 N. Crampé and A. Trombettoni, Quantum spins on star graphs and the Kondo model, Nucl. Phys. B 871, 526 (2013).

23 D. Giuliano, P. Sodano, A. Tagliacozzo, and A. Trombettoni, From four- to two-channel Kondo effect in junctions of xy spin chains, Nucl. Phys. B 909, 135 (2016).

24 D. Giuliano and P. Sodano, Realization of a twochannel Kondo model with Josephson junction networks, EPL (Europhysics Letters) 103, 57006 (2013).

25 D. Giuliano and P. Sodano, Y-junction of superconducting Josephson chains, Nucl. Phys. B 811, 395 (2009).

${ }^{26}$ F. Buccheri, G. D. Bruce, A. Trombettoni, D. Cassettari, H. Babujian, V. E. Korepin, and P. Sodano, Holographic optical traps for atom-based topological Kondo devices, New Journal of Physics 18, 075012 (2016).

27 B. Beri and N. R. Cooper, Topological Kondo effect with Majorana fermions, Phys. Rev. Lett. 109, 156803 (2012).

28 A. Altland and R. Egger, Multiterminal CoulombMajorana junction, Phys. Rev. Lett. 110, 196401 (2013).

29 A. Altland, B. Beri, R. Egger, and A. M. Tsvelik, Multichannel Kondo impurity dynamics in a Majorana device, Phys. Rev. Lett. 113, 076401 (2014).

30 A. Rahmani, C.-Y. Hou, A. Feiguin, M. Oshikawa, C. Chamon, and I. Affleck, General method for calculating the universal conductance of strongly correlated junctions of multiple quantum wires, Phys. Rev. B 85, 045120 (2012).

31 I. Affleck and D. Giuliano, Topological superconductor-Luttinger liquid junctions, J. Stat. Mech. Theory Exp. 2013, P06011 (2013).

32 D. Giuliano and I. Affleck, Real fermion modes, impurity entropy, and nontrivial fixed points in the phase diagram 
of junctions of interacting quantum wires and topological superconductors, Nucl. Phys. B 944, 114645 (2019).

33 C. L. Kane, D. Giuliano, and I. Affleck, Equivalent critical behavior of a helical point contact and a twochannel Luttinger liquid-topological superconductor junction, Phys. Rev. Research 2, 023243 (2020).

34 J. P. Pekola and B. Karimi, Colloquium: Quantum heat transport in condensed matter systems, Rev. Mod. Phys. 93, 041001 (2021).

$\begin{array}{ccc}35 & \text { F. Franz and G. Wiedemann, } & \text { Ueber } \\ \text { die } & \text { Wärme-leitungsfähigkeit der } & \text { Metalle, }\end{array}$ Annalen der Physik 165, 497 (1853).

36 G. S. Kumar, G. Prasad, and R. O. Pohl, Experimental determinations of the Lorenz number, Journal of Materials Science 28, 4261 (1993).

37 C. L. Kane and M. P. A. Fisher, Thermal transport in a Luttinger liquid, Phys. Rev. Lett. 76, 3192 (1996).

${ }^{38}$ C. L. Kane and M. P. A. Fisher, Quantized thermal transport in the fractional quantum Hall effect, Phys. Rev. B 55, 15832 (1997).

39 A. Lavasani, D. Bulmash, and S. Das Sarma, Wiedemann-Franz law and Fermi liquids, Phys. Rev. B 99, 085104 (2019).

40 B. Kubala, J. König, and J. Pekola, Violation of the Wiedemann-Franz law in a single-electron transistor, Phys. Rev. Lett. 100, 066801 (2008).

41 D. B. Karki, Coulomb blockade oscillations of heat conductance in the charge Kondo regime, Phys. Rev. B 102, 245430 (2020).

42 D. Majidi, M. Josefsson, M. Kumar, M. Leijnse, L. Samuelson, H. Courtois, C. B. Winkelmann, and V. F. Maisi, Quantum confinement suppressing electronic heat flow below the Wiedemann-Franz law (2021), arXiv:2106.06229 [cond-mat.mes-hall].

43 V. V. Ponomarenko, Renormalization of the onedimensional conductance in the Luttinger-liquid model, Phys. Rev. B 52, R8666 (1995).

44 I. Safi and H. J. Schulz, Transport in an inhomogeneous interacting one-dimensional system, Phys. Rev. B 52, R17040 (1995).

45 D. L. Maslov and M. Stone, Landauer conductance of Luttinger liquids with leads, Phys. Rev. B 52, R5539 (1995).

${ }^{46}$ P. Nozières, A Fermi-liquid description of the Kondo problem at low temperatures, Journal of Low Temperature Physics 17, 31 (1974).

47 D. Boese and R. Fazio, Thermoelectric effects in Kondo-correlated quantum dots, Europhysics Letters (EPL) 56, 576 (2001).

48 T. A. Costi and V. Zlatic, Thermoelectric transport through strongly correlated quantum dots, Phys. Rev. B 81, 235127 (2010).

49 V. Balachandran, R. Bosisio, and G. Benenti, Validity of the wiedemann-franz law in small molecular wires, Phys. Rev. B 86, 035433 (2012).

50 I. Affleck and A. W. Ludwig, The Kondo effect, conformal field theory and fusion rules, Nucl. Phys. B 352, 849 (1991).

${ }^{51}$ I. Affleck and A. W. Ludwig, Critical theory of overscreened Kondo fixed points, Nucl. Phys. B 360, 641 (1991).

52 I. Affleck and A. W. W. Ludwig, Exact conformal-fieldtheory results on the multichannel Kondo effect: Singlefermion Green 's function, self-energy, and resistivity, Phys. Rev. B 48, 7297 (1993).
53 D. B. Karki and M. N. Kiselev, Quantum thermoelectric and heat transport in the overscreened Kondo regime: Exact conformal field theory results, Phys. Rev. B 102, 241402(R) (2020).

54 A. Rahmani, C.-Y. Hou, A. Feiguin, C. Chamon, and I. Affleck, How to find conductance tensors of quantum multiwire junctions through static calculations: Application to an interacting $\mathrm{Y}$ junction, Phys. Rev. Lett. 105, 226803 (2010).

55 A. Bardas and D. Averin, Peltier effect in normal-metal-superconductor microcontacts, Phys. Rev. B 52, 12873 (1995).

${ }^{56}$ G. Benenti, G. Casati, K. Saito, and R. Whitney, Fundamental aspects of steady-state conversion of heat to work at the nanoscale, Physics Reports 694, 1 (2017).

57 F. Buccheri, A. Nava, P. Sodano, R. Egger, and D. Giuliano, Violation of the WiedemannFranz law in the topological Kondo model (2021), arXiv:2108.04156 [cond-mat.mes-hall].

58 L. Herviou, K. Le Hur, and C. Mora, Many-terminal Majorana island: From topological to multichannel Kondo model, Phys. Rev. B 94, 235102 (2016).

59 F. Nichele, A. C. C. Drachmann, A. M. Whiticar, E. C. T. O'Farrell, H. J. Suominen, A. Fornieri, T. Wang, G. C. Gardner, C. Thomas, A. T. Hatke, P. Krogstrup, M. J. Manfra, K. Flensberg, and C. M. Marcus, Scaling of Majorana zero-bias conductance peaks, Phys. Rev. Lett. 119, 136803 (2017).

60 J. Chen, B. D. Woods, P. Yu, M. Hocevar, D. Car, S. R. Plissard, E. P. A. M. Bakkers, T. D. Stanescu, and S. M. Frolov, Ubiquitous non-Majorana zero-bias conductance peaks in nanowire devices, Phys. Rev. Lett. 123, 107703 (2019).

61 P. Yu, J. Chen, M. Gomanko, G. Badawy, E. P. A. M. Bakkers, K. Zuo, V. Mourik, and S. M. Frolov, NonMajorana states yield nearly quantized conductance in proximatized nanowires, Nature Physics 17, 482 (2021).

62 R. M. Lutchyn, E. P. A. M. Bakkers, L. P. Kouwenhoven, P. Krogstrup, C. M. Marcus, and Y. Oreg, Majorana zero modes in superconductor-semiconductor heterostructures, Nature Reviews Materials 3, 52 (2018).

${ }^{63}$ K. Flensberg, F. von Oppen, and A. Stern, Engineered platforms for topological superconductivity and majorana zero modes, Nature Reviews Materials 2, 944-958 (2021).

64 E. Eriksson, A. Nava, C. Mora, and R. Egger, Tunneling spectroscopy of Majorana-Kondo devices, Phys. Rev. B 90, 245417 (2014).

65 E. Eriksson, C. Mora, A. Zazunov, and R. Egger, Non-Fermi -liquid manifold in a Majorana device, Phys. Rev. Lett. 113, 076404 (2014).

66 A. Y. Kitaev, Unpaired Majorana fermions in quantum wires, Physics-Uspekhi 44, 131 (2001).

67 J. Nilsson, A. R. Akhmerov, and C. W. J. Beenakker, Splitting of a Cooper pair by a pair of Majorana bound states, Phys. Rev. Lett. 101, 120403 (2008).

68 D. Giuliano, S. Paganelli, and L. Lepori, Current transport properties and phase diagram of a Kitaev chain with longrange pairing, Phys. Rev. B 97, 155113 (2018).

69 M. P. Anantram and S. Datta, Current fluctuations in mesoscopic systems with Andreev scattering, Phys. Rev. B 53, 16390 (1996).

70 A. L. Chernyshev and A. V. Rozhkov, Heat transport in spin chains with weak spin-phonon coupling, Phys. Rev. Lett. 116, 017204 (2016). 
71 A. Lucas and S. Das Sarma, Electronic hydrodynamics and the breakdown of the Wiedemann-Franz and Mott laws in interacting metals, Phys. Rev. B 97, 245128 (2018).

72 B. Bellazzini, M. Burrello, M. Mintchev, and P. Sorba, Quantum field theory on star graphs (2008), arXiv:0801.2852 [hep-th].

73 B. Bellazzini, M. Mintchev, and P. Sorba, Quantum wire junctions breaking time-reversal invariance, Phys. Rev. B 80, 245441 (2009).

74 M. Mintchev and P. Sorba, Luttinger liquid in a non-equilibrium steady state, Journal of Physics A: Mathematical and Theoretical 46, 0950

75 B. Bellazzini, M. Mintchev, and P. Sorba, Bosonization and scale invariance on quantum wires, J. Phys. A Math. Theor. 40, 2485 (2007).

76 Our parametrization differs by the one in [73] by a redefinition $\theta \rightarrow \pi / 3-\theta$.

77 B. Bellazzini, P. Calabrese, and M. Mintchev, Junctions of anyonic Luttinger wires, Phys. Rev. B 79, 085122 (2009).

78 S. Das and S. Rao, Duality between normal and superconducting junctions of multiple quantum wires, Phys. Rev. B 78, 205421 (2008).

79 J. S. Meyer and M. Houzet, Conductance quantization in topological Josephson trijunctions, Phys. Rev. B 103, 174504 (2021).

80 D. N. Aristov and P. Wölfle, Transport properties of a $\mathrm{Y}$ junction connecting Luttinger liquid wires, Phys. Rev. B 84, 155426 (2011).

81 D. N. Aristov, I. V. Gornyi, D. G. Polyakov, and P. Wölfle, $\mathrm{Y}$ junction of Luttinger liquid wires out of equilibrium, Phys. Rev. B 95, 155447 (2017).

82 A. Tokuno, M. Oshikawa, and E. Demler, Dynamics of one-dimensional Bose liquids: Andreev-like reflection at $\mathrm{Y}$ junctions and the absence of the Aharonov-Bohm effect, Phys. Rev. Lett. 100, 140402 (2008).

${ }^{83}$ L. Fidkowski, J. Alicea, N. H. Lindner, R. M. Lutchyn, and M. P. A. Fisher, Universal transport signatures of Majorana fermions in superconductor-Luttinger liquid junctions, Phys. Rev. B 85, 245121 (2012).

84 I. Affleck and D. Giuliano, Screening clouds and Majorana fermions, Journal of Statistical Physics 157, 666 (2014).

85 B. Beri, Majorana-Klein hybridization in topological superconductor junctions,
Phys. Rev. Lett. 110, 216803 (2013).

${ }^{86}$ F. Buccheri, H. Babujian, V. E. Korepin, P. Sodano, and A. Trombettoni, Thermodynamics of the topological Kondo model, Nucl. Phys. B 896, 52 (2015).

87 J. Cardy, Scaling and Renormalization in Statistical Physics, Cambridge Lecture Notes in Physics (Cambridge University Press, 1996).

88 D. Giuliano, D. Rossini, and A. Trombettoni, From Kondo effect to weak-link regime in quantum spin- $\frac{1}{2}$ spin chains, Phys. Rev. B 98, 235164 (2018).

89 I. Affleck, M. Oshikawa, and H. Saleur, Quantum brown(i2013)otion on a triangular lattice and boundary conformal field theory, Nucl. Phys. B 594, 535 (2001).

90 K. Michaeli, L. A. Landau, E. Sela, and L. Fu, Electron teleportation and statistical transmutation in multiterminal Majorana islands, Phys. Rev. B 96, 205403 (2017).

91 A. Zazunov, F. Buccheri, P. Sodano, and R. Egger, $6 \pi$ Josephson effect in Majorana box devices, Phys. Rev. Lett. 118, 057001 (2017).

${ }^{92}$ F. Buccheri, R. Egger, R. G. Pereira, and F. B. Ramos, Quantum spin circulator in $\mathrm{Y}$ junctions of Heisenberg chains, Phys. Rev. B 97, 220402(R) (2018).

93 F. Buccheri, R. Egger, R. G. Pereira, and F. B. Ramos, Chiral $\mathrm{Y}$ junction of quantum spin chains, Nucl. Phys. B 941, 794 (2019).

94 D. Giuliano and P. Sodano, Competing boundary interactions in a Josephson junction network with an impurity, Nucl. Phys. B 837, 153 (2010).

$95 \mathrm{H}$. Yi and C. L. Kane, Quantum brownian motion in a periodic potential and the multichannel Kondo problem, Phys. Rev. B 57, R5579 (1998).

96 C. L. Kane, M. P. A. Fisher, and J. Polchinski, Randomness at the edge: Theory of quantum Hall transport at filling 2/3, Phys. Rev. Lett. 72, 4129 (1994).

97 G. E. Blonder, M. Tinkham, and T. M. Klapwijk, Transition from metallic to tunneling regimes in superconducting microconstrictions: Excess current, charge imbalance, and supercurrent conversion, Phys. Rev. B 25, 4515 (1982).

98 G. Campagnano, P. Lucignano, and D. Giuliano, Chirality and current-current correlation in fractional quantum Hall systems, Phys. Rev. B 93, 075441 (2016). 